BNL-111770-2016-JA

\title{
CYCLOTRONS AND FFAG ACCELERATORS AS DRIVERS FOR ADS
}

\author{
LUCIANO CALABRETTA \\ Laboratori Nazionali del Sud, Istituto Nazionale di Fisica Nucleare, Via Santa Sofia 62 \\ Catania, 95123, Italy \\ calabretta@Ins.infn.it \\ FRANCOIS MEOT \\ Brookhaven National Laboratory \\ Upton, NY 11973, USA \\ fmeot@bnl.gov
}

\begin{abstract}
This review summarizes projects and studies of circular accelerators proposed to drive subcritical reactors. The early isochronous cyclotron cascades, proposed about twenty years ago, and the evolution of these layouts up to the most recent solutions based on cyclotrons and Fixed Field Alternating Gradient accelerators are here reported. The newest ideas and their prospects for development will be discussed.
\end{abstract}

Keywords: Cyclotrons, FFAG, High Power, ADS.

\section{Introduction}

Ideas, studies, and research projects on Accelerator Driven Systems (ADS) as proposed over the last 20 years are reviewed in this paper. A turning point on the ADS concept occurred in 1995, when Prof. Carlo Rubbia invented and published the concept of an Energy Amplifier (EA) [1,2]. In the simplest description the EA is a subcritical reactor driven by a fast neutron flux (energies greater than thermal reactor neutrons) produced by a high power proton beam striking a spallation target within the reactor core, with the proton beam generated by a highcurrent, GeV-energy accelerator.

The idea to use powerful accelerators to transmute nuclei has been under investigation since the early 1950s. One example is the Material Test Accelerator project [3] at Berkeley, with the objective of producing fissile material using a $350 \mathrm{MeV}, 500 \mathrm{~mA} \mathrm{CW}$ deuteron linac. This project was terminated in 1952 when plentiful uranium ore was discovered in the USA. In the late seventies, Russian scientists tried to demonstrate the concept of accelerator breeding or fuel regeneration using the Dubna accelerators [4]. In 1992 Bowman [5] proposed using a high power accelerator to produce a thermal flux of neutrons of $10^{16} \mathrm{n} / \mathrm{cm}^{2} \mathrm{~s}$, to drive a subcritical reactor, to transmute the long term high level waste, and the higher actinide waste produced in commercial reactors. These projects require very high beam current, around $100 \mathrm{~mA}$ of proton with energy 1-1.6 GeV, to achieve a positive balance between produced and dissipated power. These early projects were not successful for various reasons.

Rubbia's paper showed how to use a fast neutron flux to control the criticality of a subcritical reactor to produce much more energy than required to drive the accelerator and the nuclear plant. The possibility of using subcritical reactors with $k_{\text {eff }} \approx 0.98$, intrinsically safer, remains very appealing today to overcome the people's fear, especially after the accidents of Chernobyl and Fukushima. Indeed, the problems originated from self-sustained chain reactions in commercial reactors and the very long-lived species such as minor actinides are greatly reduced using the ADS concept [6]. Moreover, the EA concept allows more efficient use of uranium fuel and makes the existing large amount of thorium a possible reactor fuel. A very interesting possibility of the EA is the use of a subcritical reactor driven by an Accelerator to Transmute Waste (ATW) produced by existing 


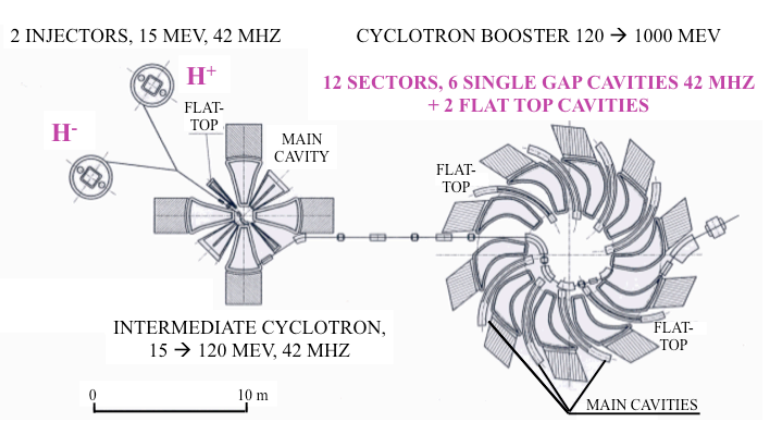

Fig. 1. Layout of the first cyclotron cascade proposed to drive the energy amplifier.

commercial reactors. The "ADS" concept includes both the ability to burn waste produced by existing reactors, and the ability to produce electricity using thorium fuel.

The main features of accelerators to be used to drive a subcritical reactor in the EA regime were given in [1]:

- $\quad$ Energy in the range 1-2 GeV;

- Beam power no less than $10 \mathrm{MW}$;

- High efficiency to convert wall plug power to beam power, $\eta>35 \%$;

- High reliability.

According to [1] the beam power required to drive a commercial subcritical reactor is greatly reduced relative to earlier projections [5], becoming to achievable with present state of the art accelerators. Rubbia's idea stimulated international interest in the accelerator community. Subsequently many major countries have an active ADS research program.

In the first part of this paper we describe ideas and solutions based on isochronous cyclotrons. In the second part we explore recent possibilities presented by the development of Fixed Field Alternating Gradient (FFAG) machines. Although isochronous cyclotrons are very different from the original FFAG concept, they can be considered a class of FFAGs that are specifically designed to work at fixed frequency; i.e. isochronous or quasiisochronous FFAGs.

In the sections 2 and 3 we describe the cyclotron solution as envisioned in [1], and the corresponding dynamics of high intensity beam in an isochronous cyclotron. The original cyclotrons on which the ADS concept was based, and more recent evolutions, will be presented in sections 4 and 5. Sections 6 and 7 present the FFAG principle and describe the status of perhaps the most advanced ADS recirculating accelerator project and a proposal to develop ADS based on this principle.

\section{ADS Requirements and Issues}

The idea of using a compact, highly reliable and modular cyclotron to drive an Energy Amplifier was presented in 1995 [1]. In Fig. 1 the layout of the proposed solution is shown.

The cyclotron was proposed as the driver for the energy amplifier due to the successful high current performance of the PSI isochronous ring cyclotron [7]. The PSI accelerator complex was designed in the late seventies originally as a meson factory with the goal of delivering a proton beam with a fixed energy of $590 \mathrm{MeV}$ at a power of $50 \mathrm{~kW}$. After 15 years of operational upgrades and development the original PSI cyclotron was able to deliver beam power up to $1 \mathrm{MW}$. This success was made possible by the replacement of the previous compact cyclotron with a new separated sector cyclotron, the PSI Injector II [8] in 1984, and raising the $\mathrm{RF}$ voltage of each cavity from the initial value of $400 \mathrm{kV}$ to $700 \mathrm{kV}$, in four steps from 1992 to 1995 (see Fig. 2). The outstanding performance of this cyclotron is in excellent agreement with the theoretical prediction [9], the scaling law:

$$
I_{\max }=k N^{-3}
$$

where Imax is the accelerated current, $N$ is the number of acceleration turns the beam makes in the cyclotron and $k$ is a proportionality constant.

It was evident at that time that the PSI cyclotron had the potential to increase beam current further by replacing the 4 original aluminum RF Cavities with copper ones, achieving almost $1000 \mathrm{kV}$ per cavity (see Fig. 2).

In addition to holding the present record for beam current in a recirculating accelerator this 40 year old PSI ring cyclotron, has other operational features essential to the ADS application: 


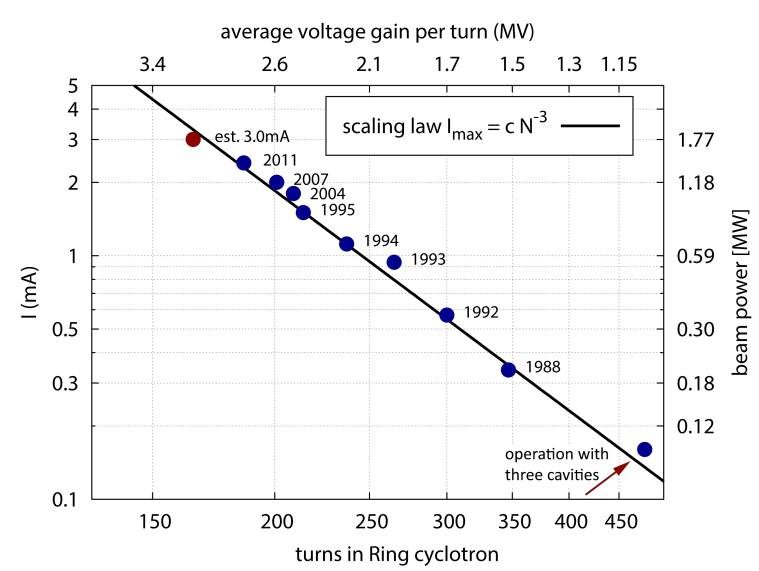

Fig. 2. The impressive growth of the PSI cyclotron beam current was mainly achieved by raising the accelerating voltage of the RF cavities.

- Continuous wave $(\mathrm{CW})$ beam;

- $90 \%$ reliability;

- High efficiency wall plug power conversion to beam power: nearly $40 \%$.

The high efficiency in transforming wall plug power to beam power is directly related to the recirculating and isochronous nature of the cyclotron, as the fixed frequency RF cavities are multi pass, used over hundreds acceleration turns (see fig.2), while in the competing linac each cavity is single pass, accelerating a proton only once, resulting in the linac being the most expansive and the largest footprint accelerator. The conversion efficiency of the PSI accelerator complex will increase further when the two flat-top cavities of PSI Injector 2 are replaced with two new accelerating cavities, thereby increasing beam power up to $1.8 \mathrm{MW}$ [10]. Since the thermal power dissipated by the RF cavities, cyclotron sectors magnets, transport line, and vacuum system does not depends on beam power delivered, the overall efficiency increases in proportion to beam power.

Fig. 3 shows a breakdown of the primary sources of accelerator failures [10] which ultimately determine the $89.5 \%$ reliability overall of scheduled beam time. This performance goes towards satisfying the requirement determined for an ADS system [11,12] in order to drive an industrial scale power generation plant. All these features promoted the choice of a cyclotron by

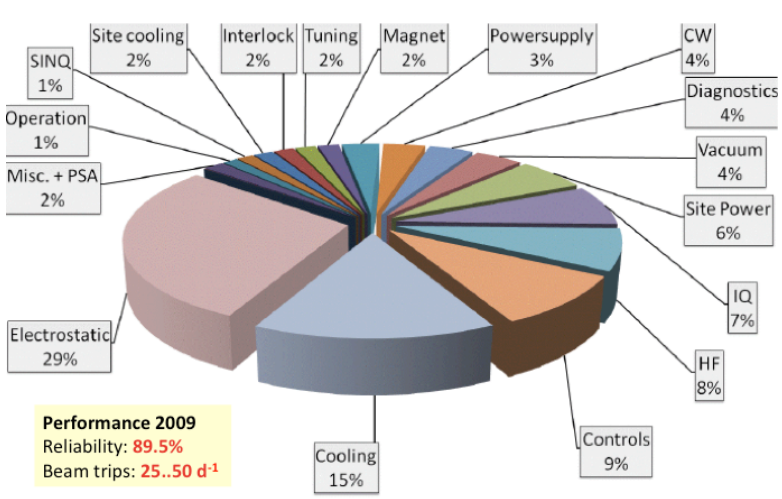

Fig. 3. The main causes of PSI down time in 2009 were: electrostatic extractor, cooling, controls and site power.

Mandrillon and Fiétier [13] as the component driver of the EA. The proposed cyclotron layout is an extrapolation of the PSI two-stage cyclotron. It consists of three cascaded cyclotron stages to deliver a $12.5 \mathrm{~mA}$ proton beam at $1 \mathrm{GeV}$ as shown in Fig. 1. The first stage accelerates the beam energy from the $100 \mathrm{keV}$ of the source to $15 \mathrm{MeV}$ at extraction. Two of these compact isochronous cyclotrons form the first stage; one accelerates protons and the other $\mathrm{H}^{-}$. The use of two injector cyclotrons allows acceleration of just $6.25 \mathrm{~mA}$ in each cyclotron to mitigate the problem of enormous space charge effects at low energy and high currents. The use of $\mathrm{H}^{-}$in one of the two injector cyclotrons allows the two beams to be merged through a stripping foil before injection into the intermediate stage a separated sector cyclotron. Acceleration in the second stage is performed by two double gap cavities, each with a constant acceleration voltage of $110 \mathrm{kV}$. To further mitigate space charge effects, two additional flat-top cavities are proposed to support a longitudinally spread-out beam bunch.

The intermediate cyclotron [14] accelerates the beam from $10 \mathrm{MeV}$ up to $120 \mathrm{MeV}$. Its design is similar to Injector II of PSI which is also equipped with two double gap RF cavities and two RF Flattop cavities. The main cavities have a voltage of about $180 \mathrm{kV}$ at the inner radii which increases up to $360 \mathrm{kV}$ at the extraction radius.

The booster stage that achieves the final energy of $1 \mathrm{GeV}$ is a scaled version of the $590 \mathrm{MeV}$ PSI spiral sector ring cyclotron, consisting of a 
12 sector with an extraction radius of less than $6 \mathrm{~m}$. The solution proposed for this final stage requires single gap RF cavities. These cavities are similar to these used in PSI ring cyclotron except they are curved rather than straight. Since this design is not a straightforward scaling of the PSI RF cavities, there are likely to be technical issues.

\section{Features of High Intensity Beam at PSI}

In this section we review the experiment and beam dynamics simulations developed at PSI during the last 40 years.

The PSI cyclotron facility was originally built to delivers an intense proton beam at an energy of $590 \mathrm{MeV}$ in order to produce intense beam of pions and muons. Today the cyclotron is mainly devoted to driving the spallation neutron source SINQ. The characteristics of the PSI accelerator complex have been described in previous papers $[7,8,10]$. Here we focus our attention on the problems of high power acceleration and how it was possible and necessary to reduce beam losses. The advantage of cyclotron is its compactness; the beam circulates inside the accelerator for many turns. On the other hand a serious limit of cyclotrons is the maximum energy achievable, which for protons is about $1 \mathrm{GeV}$ for isochronous operation, due to relativistic effects. Moreover, the beam dynamics in a cyclotron are more complicated than in a Linac: there is no phase stability. Careful tuning is necessary, and it is not possible in a conventional cyclotron design to incorporate strong focusing. This last feature produces intensity limitations due to the space charge tune shift.

An electrostatic deflector is usually used to extract protons from cyclotrons. The deflector consists of an electrode at negative potential and a septum in the form of a thin metallic electrode at ground potential placed in-between the last and the second to last turns. The electric field between the septum and the electrode deflects the outermost radial orbit at the extraction energy by a few mrad, sufficient for clean separation and extraction by a downstream magnetic septum. Often the final and penultimate orbits overlap. Particles in the overlapping region hit the septum electrode, scatter and are lost along the extraction beam line. The best way to reduce these losses is to maximize the turn-to-turn separation and simultaneously to minimize the generation of beam halo. To evaluate the step width per turn we start from the formula of the magnetic rigidity $B R$ of beam:

$$
B R=\frac{P}{q}=\sqrt{\gamma^{2}-1} \frac{m_{0} c}{q}
$$

Where: $R$ is the bending radius of a particle with momentum $P$ and charge state $q$ in a region where is a magnetic field with intensity $B ; m_{o}$ is the rest mass of the particle and $c$ the light speed and $\gamma$ the usual relativistic factor. Evaluating the logarithmic differential of the previous formula, the relation between variation of radius, magnetic field and energy of the particle beam can be obtained:

$$
\frac{d B}{B}+\frac{d R}{R}=\frac{\gamma d \gamma}{\gamma^{2}-1}
$$

Recalling that in an isochronous cyclotron the magnetic field has to satisfy the following relation:

$$
B \propto \frac{m}{q}=\frac{\gamma m_{0}}{q}
$$

According to this relation, in a magnetic field of an isochronous cyclotron the field index value is:

$$
k=\frac{R}{B_{z}} \frac{d B_{z}}{d R}=\frac{\beta}{\gamma} \frac{d \gamma}{d \beta}=\gamma^{2}-1
$$

Introducing the change of the relativistic factor $\gamma$ per turn:

$$
d \gamma / d n_{t}=U_{t} / m_{0} c^{2}
$$

Where $U_{t}$ is the energy gain per turn, the radial step width can be written as:

$$
\frac{d R}{d n}=\frac{d \gamma}{d n} \frac{d R}{d \gamma}=\frac{U_{t}}{m_{0} c^{2}} \frac{\gamma R}{\left(\gamma^{2}-1\right)(k+1)}
$$

The field index, in an isochronous cyclotron, is related to the radial tune by:

$$
k+1=v_{r}^{2}=\gamma^{2}
$$

Using this equation, (7) can be simplified and becomes: 


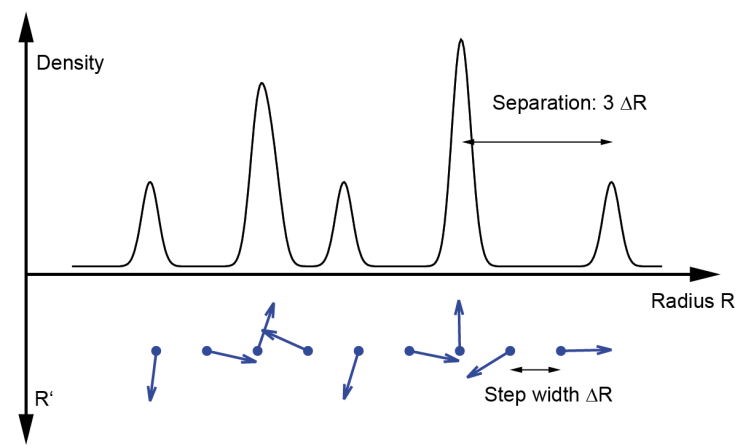

Fig. 4. Simulation of oscillations of the beam center around a closed orbit to maximize the beam separation at extraction radius. The upper plot shows the simulated beam density, while the lower half shows the clockwise rotating phase space vector of the beam centroid at each turn.

$$
\frac{d R}{d n}=\frac{U_{t}}{m_{0} c^{2}} \frac{R}{\left(\gamma^{2}-1\right) \gamma}
$$

The previous equation can be written as follows:

$$
\frac{d R}{d n}=\frac{U_{t}}{E} \frac{R}{(\gamma+1) \gamma}
$$

Where $E$ is the kinetic energy of the beam. From this formula, and the discussion below relating to beam size, it becomes evident that it is very difficult to achieve a clean extraction with an electrostatic deflector above $1 \mathrm{GeV}$. It is mandatory to maximize the energy gain per turn, and choose a large extraction radius in order to achieve a large turn separation.

Alternative ways to increase the turn separation at the extraction radius are the use of first harmonic precession and the introduction of orbit oscillations. Orbit precession can be used if $v_{\mathrm{r}}=1$, but it is difficult to use this method for cyclotrons accelerating beam at about $1 \mathrm{GeV}$. The oscillation method is convenient when the orbits have large separation, as in the PSI ring cyclotron where the beam is deliberately injected slightly off-center. Proper control of the phase and amplitude of the orbit oscillation, coupled with proper control of the behavior of the radial tune allow the beam separation be increased by a factor of three. This effect is qualitatively shown in Fig. 4.

Fig. 5 compares the simulated beam intensity

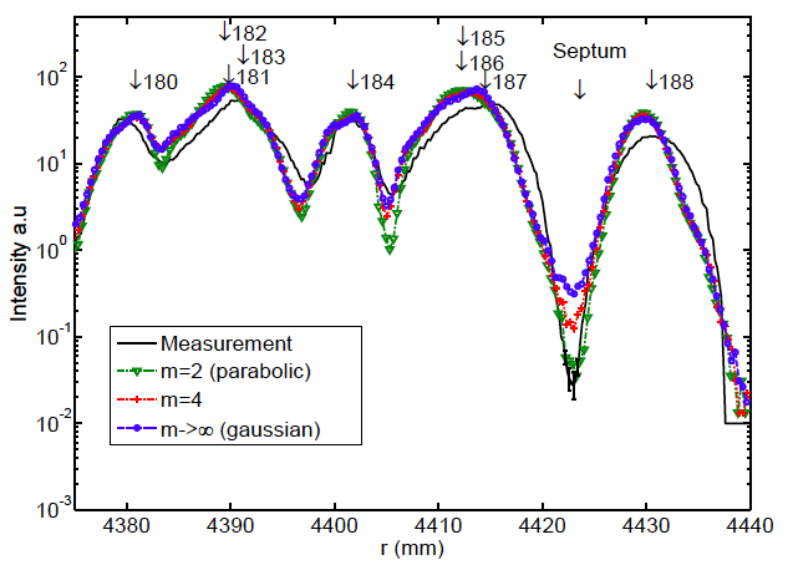

Fig. 5. Comparison among the current measure at the radial probe of the PSI Ring and beam dynamic simulations with different initial beam distributions.

versus radius $\mathrm{R}$ and the beam current measured by the radial probe in the PSI Ring cyclotron [14]. The intensity scale in Figs. 4 and 5 are linear and logarithmic respectively.

The three different beam dynamic simulations shown in Fig. 5 are obtained for different initial beam distributions. The excellent agreement between simulation and experiment is all the more impressive because the comparison is done after about $4 \mathrm{~km}$ of integration path [14].

Providing large turn separation at extraction is not sufficient to reduce the beam losses on the septum. The other key point is to suppress or to mitigate the development of beam halo. According to Joho [9], the energy spread generated by longitudinal space charge forces is transformed into transverse beam halo that produces an S-shaped beam vortex in the radial plane, see Fig. 6. Adam [15] simulated the effects of the space charge on a beam bunch accelerated in the PSI injector 2. Fig. 6 shows the result of his simulation and shows the effect of the space charge force that accelerates the particles on the leading side of the beam bunch and decelerates the particles trailing the bunch. The particles with higher energy then circulate at a larger radius and particles with lower energy circulate at a smaller radius relative to the main bunch with smaller radius. As demonstrated by W. Joho [9] the accumulated energy spread $\Delta \mathrm{E}_{\mathrm{sc}}$, in a 
cyclotron, is related to the turn number $n_{t}$ along the acceleration process by:

$$
\Delta E_{s c} \approx 2.800[\Omega] \times \frac{I_{\text {peak }} n_{t}^{2}}{\beta_{\max }}
$$

Where: $I_{\text {peak }}$ is the peak current in the bunch and $\beta_{\max }$ is the relativistic parameter at the maximum energy of the beam. The turn separation is proportional to $U_{t}$, and inversely proportional to the number of turns. Thus, the ratio between the radial beam size and the inter-turn separation is proportional to the third power of the turn number.

This analysis is consistent with Joho's relationship (1), implying that cleaner turn separation will lead to lower beam losses at extraction, and (as maximum current is ultimately limited by the power dissipated by lost beam) to higher extracted currents. Indeed, the maximum beam power lost in a cyclotron accelerator must stay below $200 \mathrm{~W}$ to allow the usual maintenance operations. Fig. 2 shows clearly that by reducing the number of turns, through increasing the energy gain per turn $U_{t}$, extracted current increased dramatically. The major improvements were achieved by upgrading the RF power amplifier and installing new copper resonators with higher $\mathrm{Q}$ and higher energy gain. These improvements led to the
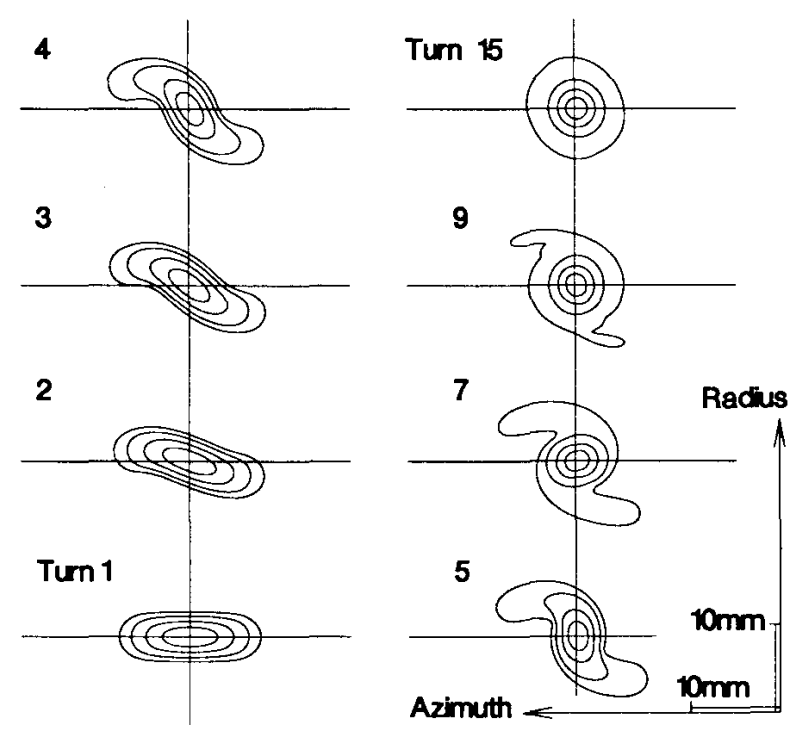

Fig. 6. Simulation of the evolution on the first few turns in the PSI injector 2 cyclotron of a $1 \mathrm{~mA}$ beam. The assumed initial bunch phase width is of $15^{\circ} \mathrm{RF}$ [15]. ability to extract higher currents while maintaining total beam losses inside the Ring cyclotron well below the operational limit requirement of $200 \mathrm{~W}$. Higher losses would complicated, or compromise, the maintenance operations of the accelerator.

The present technical limit to further beam current increases is the voltage in the flattop resonator, which cannot be raised due to thermal limitations. The $4 \mathrm{RF}$ cavities can be raised in voltage, but operational experience shows that the flattop resonator must also be raised, in proportion, to keep the beam halo acceptably low.

Important studies and simulations have been performed in recent years by Baumgarten [16] indicating that proper matching of the beam shape needs to occur in all three dimensions (radial, axial and longitudinal) to achieve higher stable beam currents and to suppress beam blowup and losses.

According to the operational experience it is evident that, unlike the ring cyclotron, the PSI injector II can deliver much more beam operating the Flat-top cavities just to produce acceleration. A plan to replace these cavities with new accelerating cavities is in progress [10]. A new concept for a better and different use of the flattop cavities to achieve the proper matching of the beam, in all three dimension [16], is under way, but further beam dynamics simulation and comparison with experimental data are mandatory to validate the new concepts and codes.

Although the ultimate intensity achievable by cyclotrons scaling from the PSI experience is not exact, an estimated limit is 5 to $10 \mathrm{~mA}$.

\section{Early ADS Cyclotron Proposals}

Spurred by the work of Rubbia at CERN $[1,2]$, several cyclotron-based ADS projects were subsequently proposed.

\subsection{The Dream Machine}

In 1996, Stammbach presented an alternative ring cyclotron [17] (see Fig. 7), similar to the PSI machine. This ring cyclotron, also called the "Dream Machine", is a 12-sector machine with eight accelerating RF cavities and two flat-top 


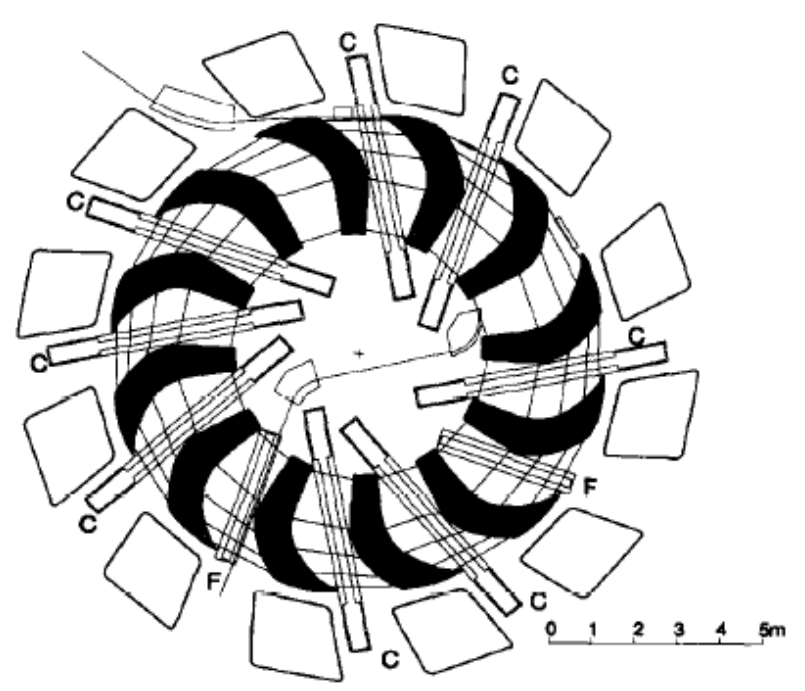

Fig. 7. Layout of the so-called "Dream Machine": $10 \mathrm{~mA}$ proton beam at $1 \mathrm{GeV}$, proposed by T. Stammbach [17].

cavities. It is quite similar to the cascade proposed in [13] and shown in Fig. 1. The main difference is that the RF cavities are straight and not bent. Another difference is that the proton beam produced by the ion source is accelerated by an electrostatic platform to a kinetic energy of $1 \mathrm{MeV}$ and then injected into a four-sector cyclotron that increases the beam energy to $120 \mathrm{MeV}$. According to [17], the expected efficiency for transforming wall plug power to the $10 \mathrm{MW}$ of beam power is between $40 \%$ and $45 \%$. This takes into account also the $4 \mathrm{MW}$ of power consumed by injector, preinjector, magnets, and vacuum pumps.

\subsection{The Negatively charged $H$ ions}

An alternative solution based on the acceleration of $\mathrm{H}^{-}$(an ion consisting of a proton and two electrons) was proposed by Craddock to overcome the problems related to the extraction of high power beams of protons [18]. According to experience at the TRIUMF, a single stage cyclotron able to accelerate $\mathrm{H}^{-}$ions is appealing for the following reasons. Extraction is performed using a stripper foil that provides simple and reliable high extraction efficiency, better than $99.9 \%$.

Energy gain per turn requirement is reduced significantly, since well separated orbits are not necessary. Operation with lower voltage increases the conversion efficiency from wall plug power to beam power. Changing the radial position of the stripper easily varies the extraction energy.

Unfortunately this solution has serious drawbacks. The small binding energy of the second electron in the $\mathrm{H}^{-}$ion (about $0.7 \mathrm{eV}$ ) poses a serious limit to the maximum magnetic field. To accelerate $\mathrm{H}^{-}$to 800 or $1000 \mathrm{MeV}$ and to ensure acceptable electromagnetic (Lorentz) stripping losses lower than $50 \mathrm{~W}$, the maximum usable fields are $0.378 \mathrm{~T}$ and $0.326 \mathrm{~T}$, respectively. Consequently the extraction radii are 16.1 and $21.7 \mathrm{~m}$. Moreover, the vacuum has to be very good, better than $10^{-6} \mathrm{~Pa}$. Finally, the amount of thermal power deposited on the stripper foil by the removed electrons is about $10 \mathrm{~kW}$. This is very large and significantly reduces the average lifetime of stripper foils even in the perspective of using multiple stripper foils.

The stripping extraction of $\mathrm{H}^{-}$is a two step process. Neutral hydrogen particles are produced, with at least a $0.01 \%$ probability. The associated beam loss, equivalent to about $1 \mathrm{~kW}$ of power inside the machine seriously limits the maintenance accessibility (The nominal accepted limit is about $200 \mathrm{~W}$ ).

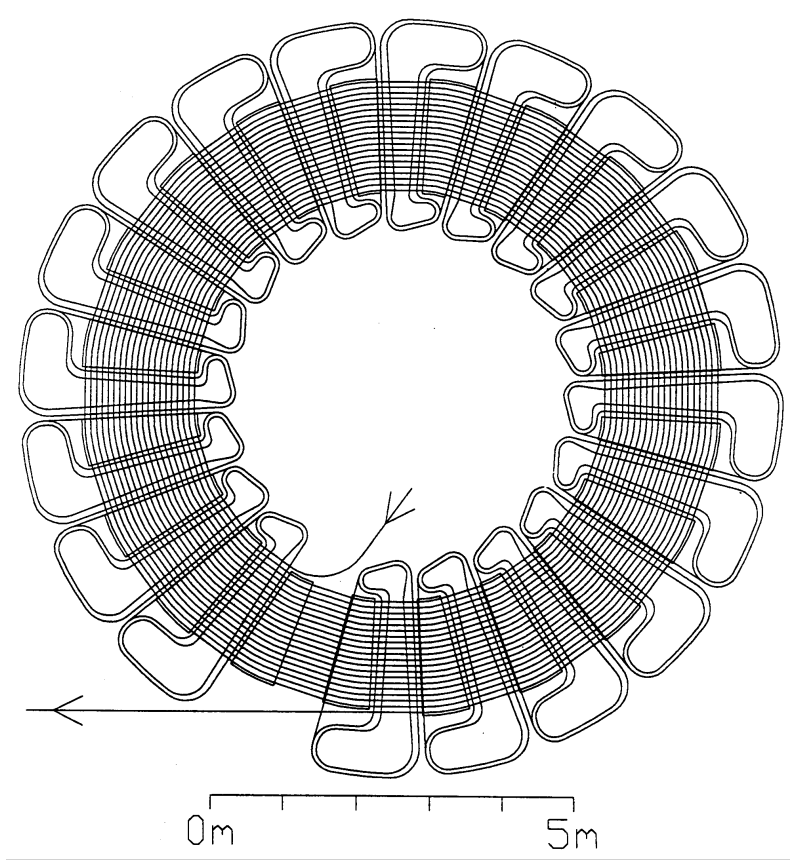

Fig. 8. Layout of a large separated orbit cyclotron. 


\subsection{Separated orbit cyclotron}

The use of a separated-orbit cyclotron (SOC) as an ADS driver was presented by Trinks, see Fig. 8, at the 1997 Santa Fe workshop [19,20]. More details on the SOC are discussed in section 5.3 of this review.

\subsection{Molecular hydrogen $\mathrm{H}_{2}^{+}$}

A proposal to use a compact superconducting cyclotron to accelerate the molecular hydrogen beam $\mathrm{H}_{2}{ }^{+}$, consisting of two protons bound by an electron, was proposed in 1997 at the European Cyclotron Progress Meeting held at KVI (NL).

According to this idea, cyclotrons able to reach energies up to $110 \mathrm{MeV} / \mathrm{amu}$ were proposed were proposed [21,22]. The advantage of accelerating $\mathrm{H}_{2}{ }^{+}$is that a stripper foil inserted in the last accelerated orbit, enables the protons emerging from the stripper to escape from the cyclotron field without any further active elements. Fig. 9 shows different proton trajectories for a broad range of initial tangential angles. The azimuthal position of the stripper can be used to select the trajectory that minimizes the beam envelope.

A similar superconducting cyclotron [23] was proposed to replace the first and second cyclotron of the cascade accelerator complex shown in Fig. 1. In the following years the advantages of using $\mathrm{H}_{2}{ }^{+}$ to produce a $10 \mathrm{MW}$ proton beam became clear, and stimulated the development of further proposals.

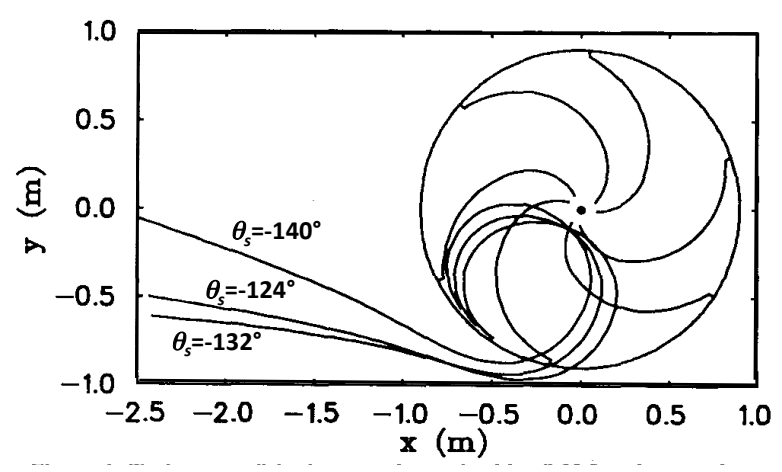

Fig. 9. Trajectories of proton beam extracted by stripping from a $\mathrm{H}_{2}{ }^{+}$accelerated beam produced by stripper foil placed at different angular positions.

\subsection{Advantages of molecular hydrogen $\mathrm{H}_{2}^{+}$}

The main advantage of accelerating singly charged molecular hydrogen, $\mathrm{H}_{2}{ }^{+}$is that extraction from the ring cyclotron could be accomplished by stripping, with virtually no losses. Stripping can also be used if the orbits are too close or overlapping. Turn separation becomes irrelevant, because all ions will pass through the foils, and all will be stripped. This method is used at the TRIUMF cyclotron and also in hundreds of commercial cyclotrons. According to experience with commercial cyclotrons, two stripper foils can be used at the same time to distribute the load over two separate targets. Splitting the beam between two foils also reduces thermal heating.

Another advantage of $\mathrm{H}_{2}{ }^{+}$compared to protons is the reduction of space charge effects at the low energies of the injected beams. The generalized perveance $K$ (see Reiser [24]) gives a measure of the space charge intensity:

$$
K=\frac{q I}{2 \pi \epsilon_{0} m \gamma^{3} \beta^{3}}
$$

According to this equation, a $5 \mathrm{~mA} \mathrm{H}_{2}{ }^{+}$beam of

Another advantage of $\mathrm{H}_{2}^{+}$compared to protons is the reduction of space charge effects at the low energies of the injected beams. The generalized perveance $K$ (see Reiser [24]) gives a measure of the space charge intensity:

$$
K=\frac{q I}{2 \pi \epsilon_{0} m \gamma^{3} \beta^{3}}
$$

According to this equation, a $5 \mathrm{~mA} \mathrm{H}_{2}^{+}$beam of $70 \mathrm{keV}$ has a space charge similar to a $2 \mathrm{~mA}$ proton beam with $30 \mathrm{KeV}$. Proton beams with $2 \mathrm{~mA}$ current have been already been accelerated, in the central region of commercial cyclotrons [25].

The great advantage with respect to the acceleration of $\mathrm{H}^{-}$is that, in order to electromagnetically dissociate the $\mathrm{H}_{2}{ }^{+}$, at the same $\beta \gamma$, a magnetic field about 10 times higher is necessary. The electron in the $\mathrm{H}_{2}^{+}$molecule in its ground state is considerably more tightly bound than in the $\mathrm{H}^{-}$ion $(2.8 \mathrm{eV}$ instead of $0.7 \mathrm{eV})$ and the overall size of the $\mathrm{H}_{2}^{+}$ion is smaller than $\mathrm{H}^{-}$. Consequently, magnetic field as high as $5 \mathrm{~T}$ are usable to accelerate $\mathrm{H}_{2}{ }^{+}$up to $1 \mathrm{GeV}$. Despite 


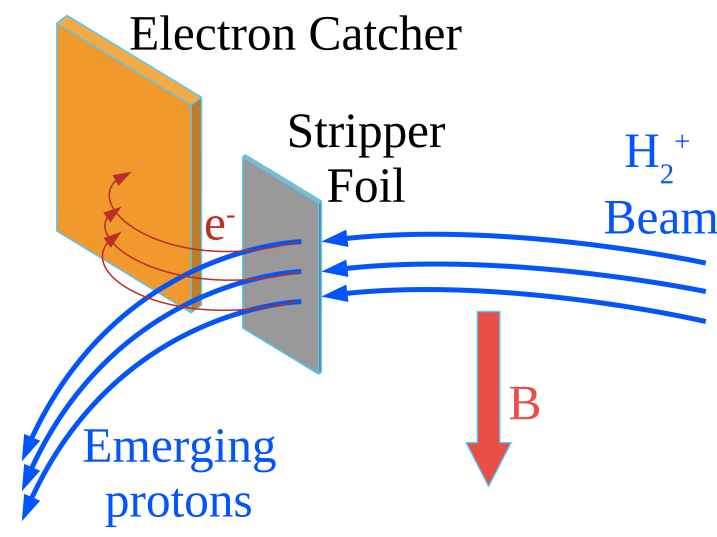

Fig. 10. Trajectories of the $\mathrm{H}_{2}^{+}$ions, of proton and of the electron removed by the stripper foil. The electrons catcher is placed at an outer radius beyond the last accelerated orbit.

having twice the magnetic rigidity the radius of a superconducting ring cyclotron is similar or smaller than in the case of cyclotron like the "Dream Machine".

Furthermore, the number of electrons produced at the stripper for each proton extracted through $\mathrm{H}_{2}{ }^{+}$dissociation is 4 times smaller than for $\mathrm{H}^{-}$. The smaller number of electrons reduces the heating of the stripper foil. Moreover, if the stripper foil is placed in a region where the magnetic field is lower than $2 \mathrm{kGauss}$ and if the $\mathrm{H}_{2}{ }^{+}$has energy higher than $800 \mathrm{MeV} / \mathrm{amu}$, the bending radius of the removed electrons is about $12 \mathrm{~mm}$, and it is possible to stop the electrons produced by the stripper in a protection catcher. This is illustrated in Fig. 10, where the magnetic field bends the $\mathrm{H}_{2}{ }^{+}$and the protons around the center of the cyclotron, while the electrons are bent toward the outer radii. In this way it is possible to stop the electrons on a catcher placed at a radius larger than the extraction orbit and minimize the heating of the stripper foil.

\section{Three New Superconducting Cyclotron Proposals}

The common feature of the three new projects proposed is the use of superconducting technology, and a final energy of around $800 \mathrm{MeV} / \mathrm{amu}$. The first project, studied by the Catania group [26], is a two-stage cyclotron complex proposed to drive the DAESALUS experiment [27] that plans to measure

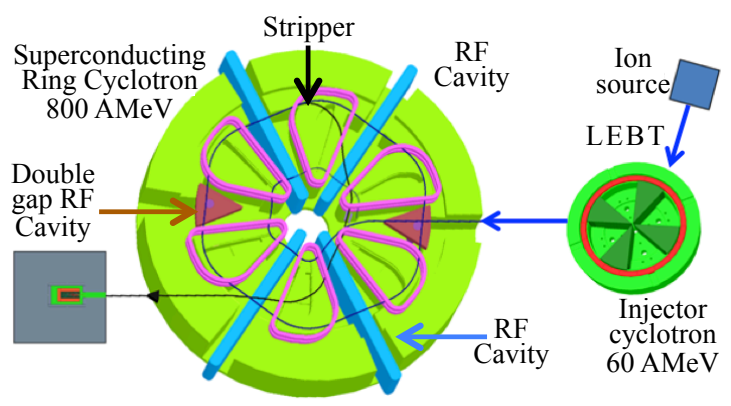

Fig. 11. Layout of DAESALUS two stage cyclotron complex. The SRC is equipped with four single gap RF cavities and two double gap cavities (brown). The stripper position and the extraction trajectories are also shown.

the $\mathrm{CP}$-violation parameter $\delta_{\mathrm{CP}}$ in the neutrino sector. The second is the stacked-cyclotron layout developed by P. McIntyre [28] and his coworkers, and the third is the single-stage superconducting cyclotron for $\mathrm{H}_{2}^{+}$proposed by P. Mandrillon [29].

\subsection{MeV/amu DAE $\delta A L U S$ cyclotron}

The most recent $800 \mathrm{MeV} / \mathrm{amu}$ Superconducting Ring Cyclotron (SRC) proposed for the DAESALUS experiment by Catania group is a further development of a previous proposal presented at EPAC in 2000 [30].

The new proposal consists of two coupled cyclotrons. The first is a room temperature cyclotron able to accelerate $\mathrm{H}_{2}^{+}$up to $60 \mathrm{MeV} / \mathrm{amu}$. The beam is extracted from this cyclotron by an electrostatic deflector. The second stage is a six

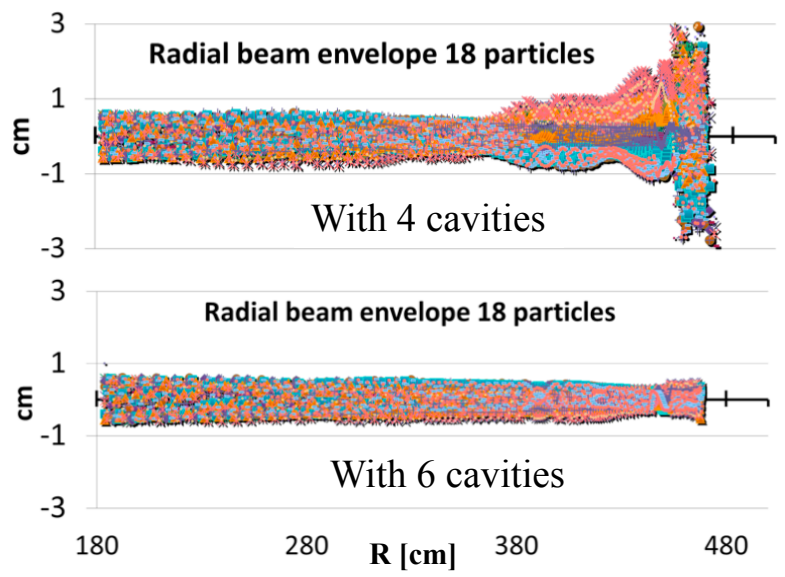

Fig. 12. Radial beam envelope vs. radius achieved with 4 accelerating cavities (top) and with 6 cavities (bottom). 


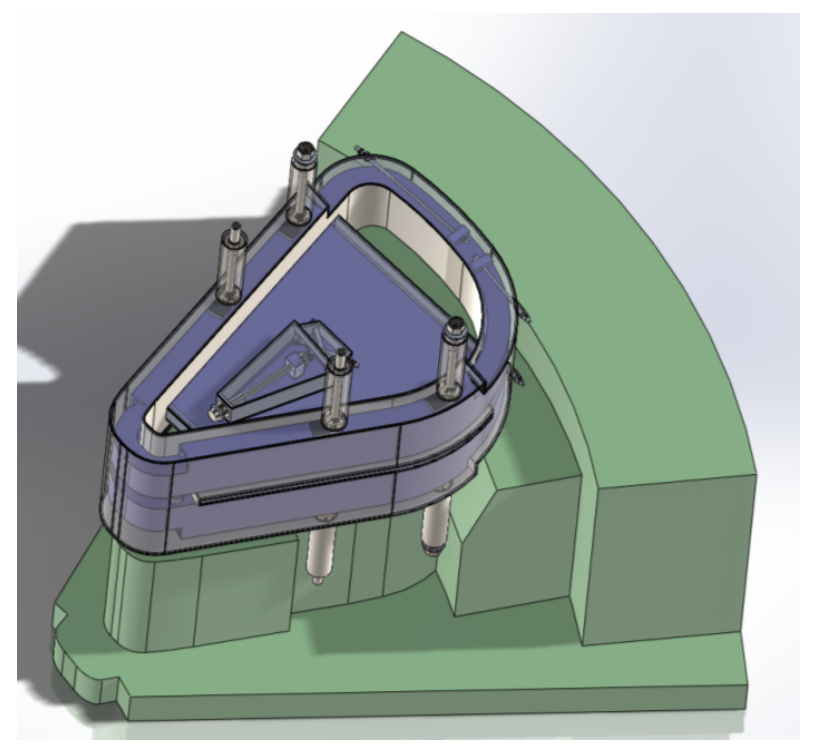

Fig. 13. One sector of the D-SRC. The lower iron, the cryostat and the tie rods to hold the superconducting coils are shown.

sector SRC able to accelerate $\mathrm{H}_{2}^{+}$up to $800 \mathrm{MeV} / \mathrm{amu}$ [26]. Fig. 11 shows the layout of this cyclotron complex. The stripper foil is placed at the entrance of a sector magnet where the field is around $0.1-0.15 \mathrm{~T}$. The proton trajectories cross the central area of the cyclotron and exit after half a turn. There is no interference with the structure of the cyclotron and only a small steering magnet placed in the central region is necessary to guide the extracted ions' trajectory, and to keep the vertical beam envelope small. The preliminary design with eight superconducting sectors was updated to a six-sector solution, in order to have enough room to install accelerating RF cavities similar to those used in the PSI ring cyclotron. The use of only 4 cavities in an SRC with 6 sectors poses a problem of uncontrolled radial and vertical beam growth when the resonance $v_{\mathrm{r}}=2$ is crossed. This problem has been removed by adding two additional double-gap RF cavities. Fig. 12. shows the radial beam envelope with and without the insertion of the two additional cavities.

The use of a reduced number of cavities ( 6 for the SRC and 8 for the Dream Machine), the absence of the 2 flattop cavities, and the use of superconducting sector magnets all increase the

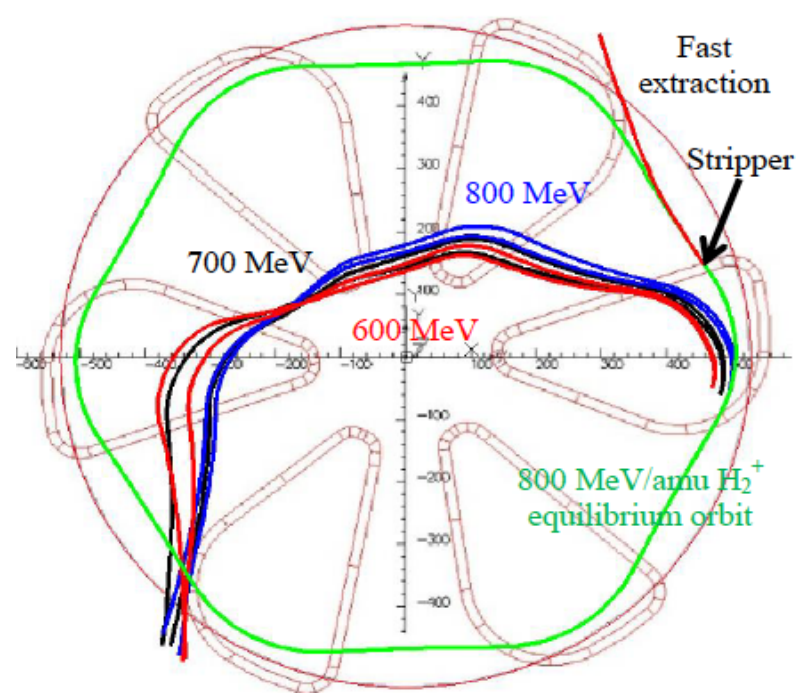

Fig. 14. Trajectories of proton with different energies produced by dissociation of the vibrational states of $\mathrm{H}_{2}^{+}$induced by localized magnetic field bumps.

conversion efficiency from wall plug power to the $10 \mathrm{MW}$ beam to $60-65 \%$.

The design of the superconducting sector magnet (see Fig. 13), was performed in collaboration with the Plasma Science and Fusion Center (PSFC) at MIT [31], and is quite similar to the RIKEN Superconducting Ring Cyclotron.

The most serious problem posed by acceleration of $\mathrm{H}_{2}^{+}$is the existence of many long-lived vibrational states. Their electron binding energies are lower than the ground state, and can be dissociated by electromagnetic stripping in the high magnetic fields. A solution to dissociate the majority of these vibrational states at the source level is highly desirable.

Alternatively, dissociation of the vibrational states is potentially possible along the matching line between the injector cyclotron and the SRC using very high-field (10-20 T) bending magnets.

A solution to remove the most dangerous of these [26] less-bound vibrational states consists of introducing a magnetic field bump of 0.3-0.5 $\mathrm{T}$ in a narrow azimuthal region of each sector of the SRC. Such a field bump with angular extension of $1^{\circ}-2^{\circ}$ can be produced by permanent magnets. Fig. 14 shows the trajectories of the protons of different energies corresponding to dissociation of different vibrational states induced by the magnetic field 
bump. All these protons can be directed and collected outside of the vacuum chamber. On the right of Fig. 14 is shown a possible fast extraction by a stripper foil, placed at the exit of the sector. This is an interesting solution alternative to that previous investigated, as shown in Fig. 11.

The fast extraction trajectory shown in Fig. 14 is achieved by artificially increasing the reversed field along the extraction path by $0.5 \mathrm{~T}$.

Beam dynamics studies of acceleration in the original 8 sector magnet design of DAESALUSSRC were made using the OPAL code $[32,33]$.

These simulations took into account space charge effects, predicting no beam losses. Even though the radial distribution of the accelerated beam could have some long tails, the proton beam has good quality. Fig. 15 shows the radial distribution and the energy spread of the proton beam emerging from the stripper.

Probably the most critical aspect of DAESALUS accelerator complex is the injection and acceleration of the $\mathrm{H}_{2}{ }^{+}$beam through the compact injector cyclotron. The OPAL code [33] was also used to simulate space-charge effects in the 4-sector compact cyclotron. A proper design of the magnetic fall off in the extraction region produces a significant increase in the turn separation. This effect is well demonstrated in Fig. 16 where the beam distribution for the last four orbits is shown.

The estimated beam power dissipated on a septum electrode $0.3 \mathrm{~mm}$ thick is about $120 \mathrm{~W}$ for an extracted $\mathrm{H}_{2}^{+}$beam of $5 \mathrm{~mA}$. Beam halo is removed using a thin stripper foil inserted between the last second and third turns. This foil strips the $\mathrm{H}_{2}{ }^{+}$in the beam halo that would strike the septum,

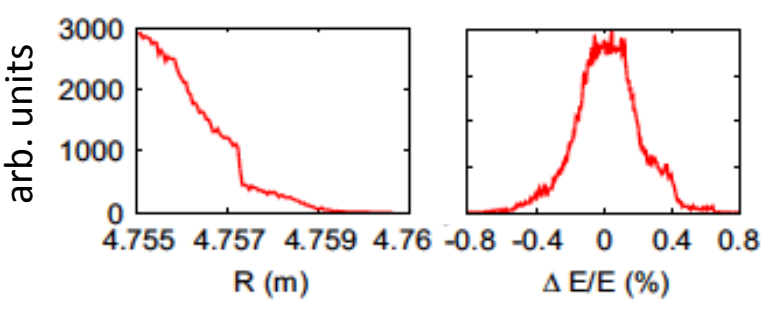

Fig. 15. Radial and energy distribution of the proton beam extracted from an 8 sectors SRC. Simulation made by OPAL code.

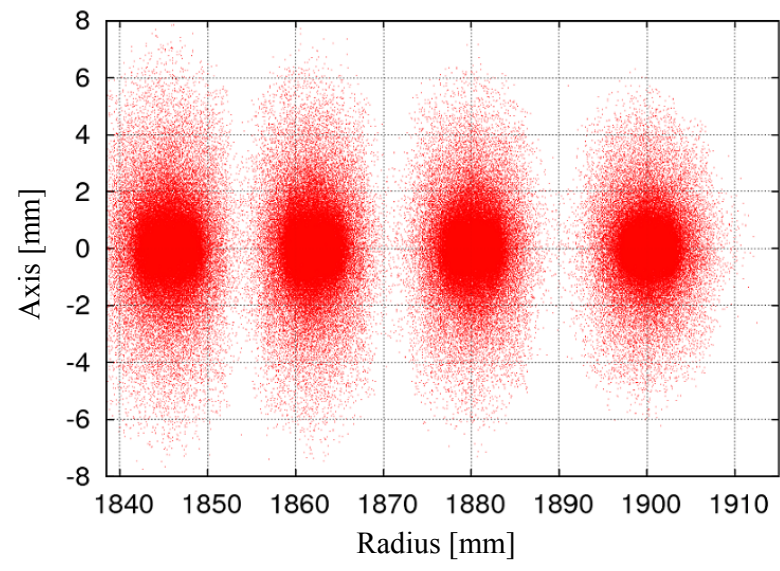

Fig. 16. Snapshot of the last 4 accelerated turns of a $60 \mathrm{MeV} / \mathrm{amu}$ injector cyclotron. It is evident how the separation between the turns increases from $14 \mathrm{~mm}$ up to $20 \mathrm{~mm}$.

and sends the stripped protons out of the cyclotron to avoid activation.

\subsection{The Stacked cyclotrons}

An early version [28] proposed to stack 5 isochronous cyclotrons into a single magnetic structure, to share the same magnetic flux. The acceleration of $10 \mathrm{~mA}$ of protons is shared among the 5 cyclotrons that have to deliver only $2 \mathrm{~mA}$ each one. This solution reduces greatly the problem of space charge but increases the complexity of the accelerator. This proposal evolved to the completely new design shown in Fig. 17, which preserve the stacked configuration and the fluxcoupled configuration [34].

The new proposal consists of a four sector injection stacked cyclotron that accelerates the proton beam to $100 \mathrm{MeV}$ (TAMU100, with an extraction radius $4.6 \mathrm{~m}$ ) and a second 12 sector stacked cyclotron to raise the energy to $800 \mathrm{MeV}$ (TAMU800, extraction radius $7.6 \mathrm{~m}$ ). An RFQ accelerator stacked is used to inject the proton beam into the TAMU100, at an energy of $2.5 \mathrm{MeV}$. The main difference with respect to the isochronous cyclotrons is in the configuration as a Strong Focusing Cyclotron (SFC). This difference is shown in Fig. 18, where the tune plot shows the acceleration path of the PSI ring cyclotron and the acceleration point for the SFC. 


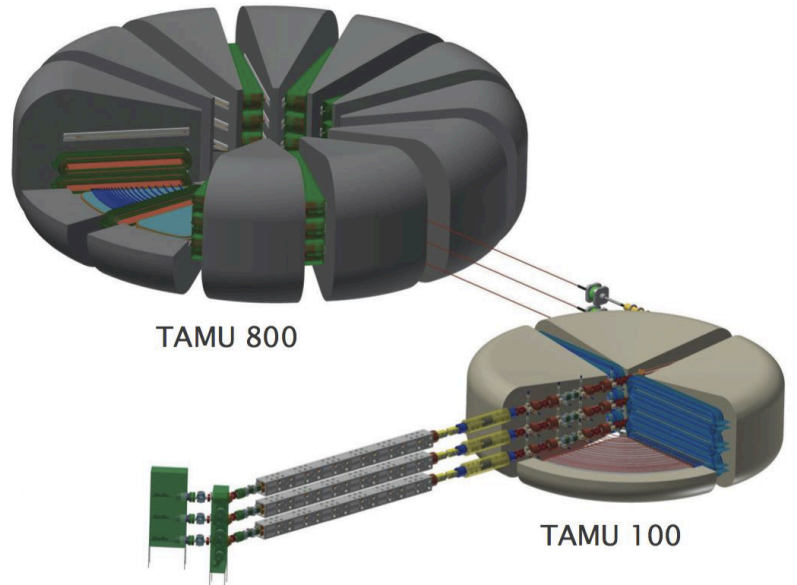

Fig. 17. Schematic view of the two stage stacked cyclotron. RFQ accelerators are used to inject the beam in the TAMU100.

The operational values $v_{\mathrm{z}}$ and $v_{\mathrm{r}}$ of SFC are about twice the maximum values of the PSI ring cyclotron. Higher focusing values mitigate space charge effects greatly. Usually an isochronous cyclotron works in the regime of weak focusing achieved by the azimuthal variation of the field and the bending of the sectors.

To achieve the SFC configuration, additional quadrupole focusing is provided within the sectors themselves. The main magnetic field is $1 \mathrm{~T}$. Isochronism is achieved by tapering the aperture of

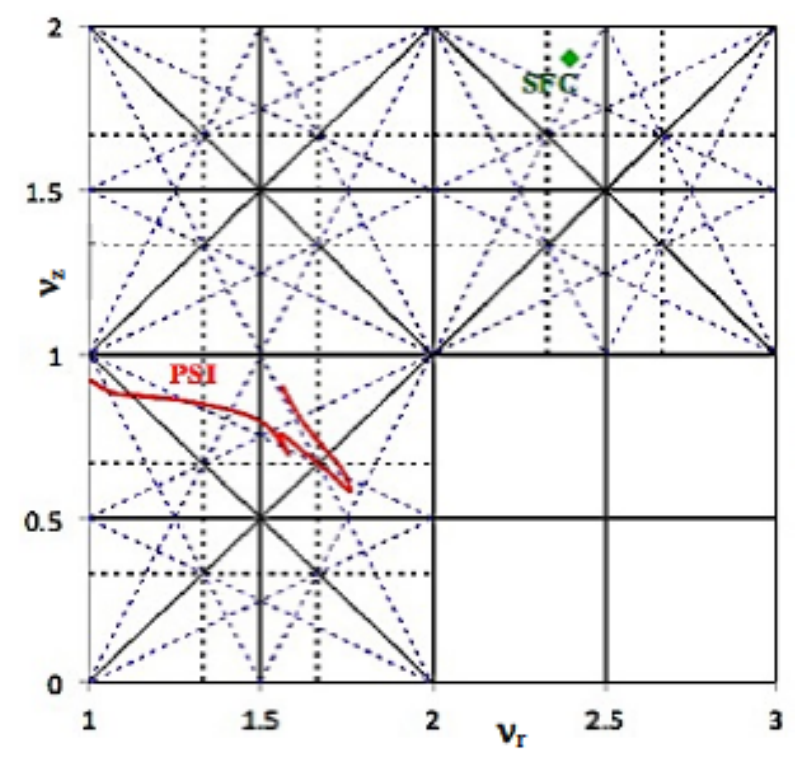

Fig. 18. Tune plot showing the acceleration path for the PSI ring cyclotron and the working point for the TAMU800.

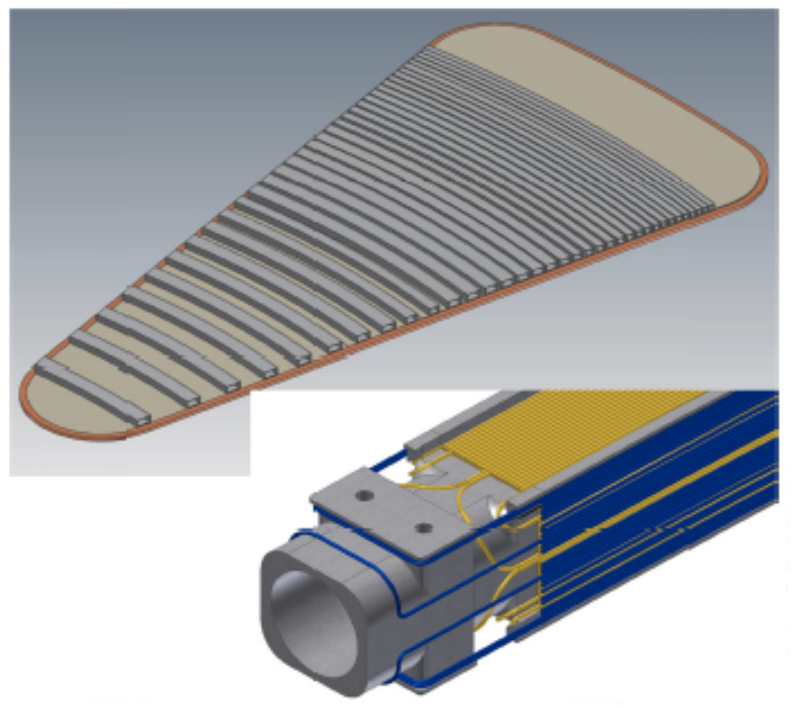

Fig. 19. Array of 35 quadrupole focusing channels inside a sector (top). Detail of one QFC (bottom) with the single layer Panofsky quadrupole (gold) and window frame dipole windings (blue).

the pole. A set of 35 Quadrupole Focusing Channels (QFC) are installed on the plate of each sector of the TAMU800. Each channel contains a Panofsky quadrupole and window frame dipole windings, as shown in Fig. 19. The QFC's in the two consecutive sectors are fed in opposite senses to guarantee a FD transport channel. The QFC's and the dipoles adjust the focusing and fine tuning of the particle orbits independently. QFC and dipole windings are made of superconducting cable $\left(\mathrm{MgB}_{2}\right)$, with a working temperature of $15-20 \mathrm{~K}$. The useful aperture for the beam in each QFC is $46 \mathrm{~mm}$.

Another great advantage of the SFC is to have sectors with straight edges that simplify the

insertion of the RF cavities.

A stacked cyclotron based on the use of the SFC recalls the concept of the Separated Orbit Cyclotron (SOC) that was proposed by Russel in 1963 [35]. Trinks built TRITRON in the 1990's specifically to investigate the feasibility and related problems of an SOC [19]. The main problem that TRITRON met was the magnetic influence between near channels. To avoid this problem the separation among the orbits in the new stacked cyclotrons is much larger than in TRITRON. This 

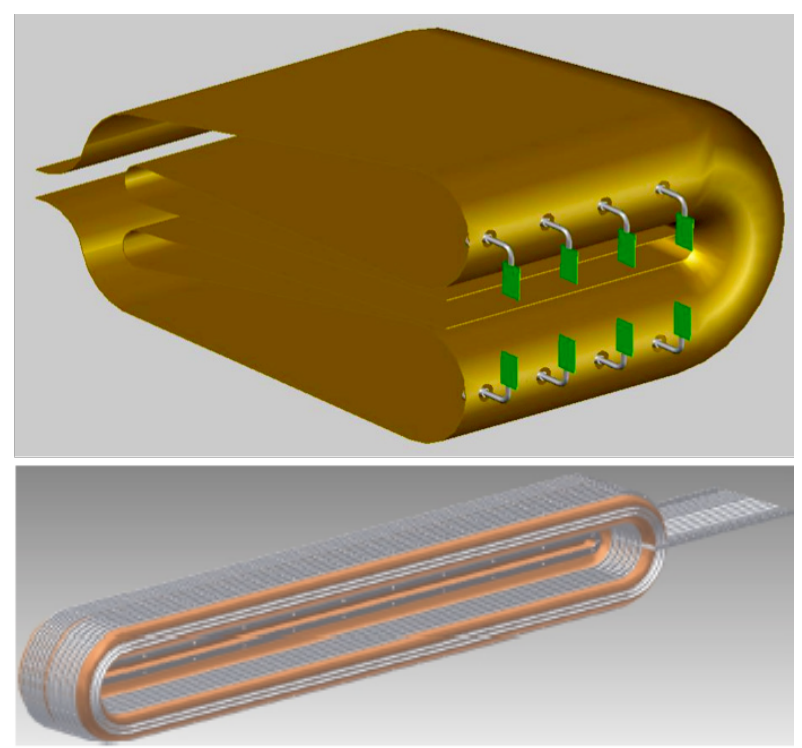

Fig. 20. Evolution of the single gap RF resonator. On top a sliced section of a $50 \mathrm{MHz} \mathrm{Cu}$ cavity. The green plates are the multiple couplers. On bottom, the new proposed $100 \mathrm{MHz}$ superconducting cavity.

requires using a large energy gain per turn. In TAMU800 this is accomplished by $10 \mathrm{RF}$ cavities operated at harmonic 19 to reduce their size to fit the space between the sectors.

An interesting product of the TAMU group is the design of a new kind of quarter wave RF cavity, evolving from PSI's single gap cavity. The top and bottom sides of the PSI cavities are folded and joined together, as shown in Fig. 20. The bottom of Fig. 20 shows the new superconducting $100 \mathrm{MHz}$ resonator [36]. Superconducting cavities would increase greatly the efficiency of an ADS cyclotron.

A further TAMU investigation feeds the RF resonator using 10 independent power couplers distributed across the length of the cavity. Each power coupler could be fed by a new generation of high efficiency solid-state amplifier able to deliver up to $50 \mathrm{~kW}$. These innovation $\mathrm{QFC}$ and superconducting cavities, need cryostats, whose design is not trivial.

Beam dynamics simulations including space charge effects of a proton beam current in excess of $10 \mathrm{~mA}$ have been performed. The results showed that the beam is well confined inside the beam pipes of the QFC. According to these positive simulations the proposers plan to operate each SFC with beam current in excess of $10 \mathrm{~mA}$. So a stacked cyclotron with 4 SFC should be able to drive 4 subcritical reactors [34].

The advantage of using a stacked cyclotron configuration becomes not relevant in the perspective to accelerate $12.5 \mathrm{~mA}$, because the stacked solution poses serious technical problem. Probably for this reason at IPAC 2015 a solution based on a single strong focusing cyclotron was presented [37,38].

However, $12.5 \mathrm{~mA}$ accelerated proton beam halo and potential beam loss in case of failure of a single RF cavity, and also source trips, could generate problems for an SOC equipped with superconducting cavities.

\subsection{Single cyclotron to accelerate $\mathrm{H}_{2}^{+}$up to $800 \mathrm{MeV/amu}$}

A single cyclotron able to accelerate $\mathrm{H}_{2}^{+}$has been proposed by Mandrillon [27] of AIMA company. It is a mix of six separated sector cyclotrons at outer radii but in the inner region it is similar to a

compact cyclotron with 6 fold symmetry. The isochronous field is produced by the six sector magnets powered by a pair of superconducting coils. Each coil is wrapped around the outer part of the six sectors but in the inner region it is bent in the opposite direction through the valley. The coils are not parallel to the median plane.

To leave room for installation of the six doublegap accelerating cavities, the upper and lower coils increase their separation in the inner regions.

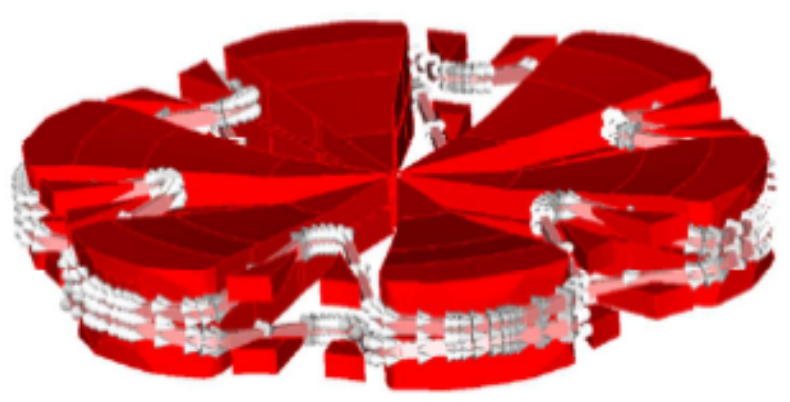

Fig. 21. View of the magnetic circuit of the single stage 800 $\mathrm{MeV} / \mathrm{amu}$ cyclotron for $\mathrm{H}_{2}{ }^{+}$. 
Fig. 21 shows the layout of the magnet circuit of the cyclotron. Beam extraction is performed by the stripper placed at the exit of the main sector, as shown in Fig. 22. Similar to Fig. 14, beam is bent toward the outer radii. The reversed magnetic field in each valley is greatly increased by adding two wedges of iron, placed near the superconducting coil, to achieve a large separation between the extracted trajectory and the last accelerated orbit, see Fig. 21. This elegant extraction method could also be used in DAESALUS ring cyclotron.

To mitigate the space charge effects three independent beams of $\mathrm{H}_{2}{ }^{+}$are injected at $60 \mathrm{keV}$, at three angular positions rotated by $120^{\circ}$ relative to one another. The average field at the center of the cyclotron is quite low, and the first orbit radius is about $12 \mathrm{~cm}$. The use of three independent sources working in parallel also reduces the problem related to beam ripple from a single source, and mitigates the risk of ion source trips a serious issue for ADS. The beam is accelerated by 6 quarter wave double-gap RF resonators working at $36.3 \mathrm{MHz}$, at harmonic 6. The resonators have a special shape to fit the valley space with the coil cryostat. The simulated quality factor $\mathrm{Q}$ of the cavity is 6200 , and the accelerating voltage rises from $150 \mathrm{kV}$ to $450 \mathrm{kV}$ between inner and outer radii. The maximum energy gain per turn is expected to be higher than $5 \mathrm{MeV}$.

This proposal also has some challenges. One is

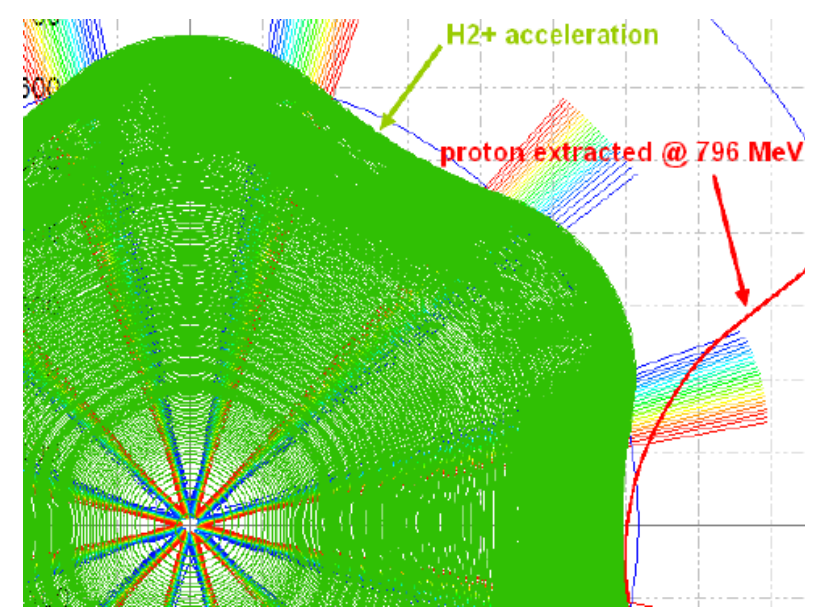

Fig. 22. Layout of the magnetic circuit of a single stage 800 $\mathrm{MeV} / \mathrm{amu}$ cyclotron for $\mathrm{H}_{2}^{+}$.

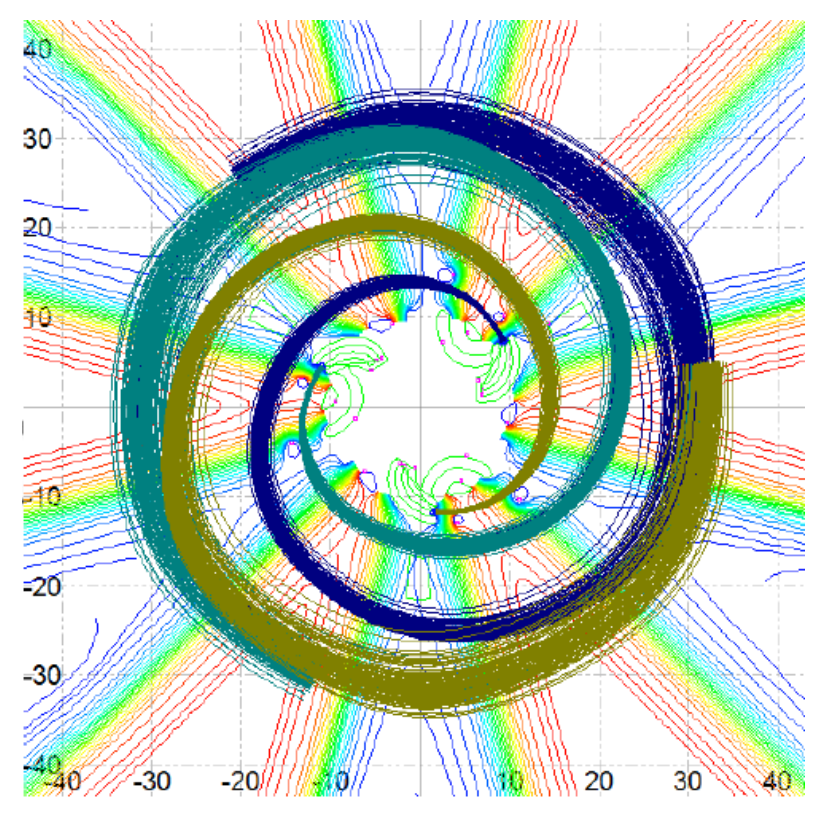

Fig. 23. Triple injection of $\mathrm{H}_{2}{ }^{+}$into the single stage cyclotron.

the complexity of the superconducting coil shape and its dimensions. Each coil is $50 \mathrm{~m}$ long, with outer and inner radii of about $6.6 \mathrm{~m}$ and $4.2 \mathrm{~m}$. The coil does not stay in a plane but is closer to the median plane at the outer radii. The bending curvature of the coil is reversed at the inner radius, a critical issue for superconducting coils.

A second issue is related to beam losses due to the interaction of the beam with residual gas in the vacuum chamber. DAESALUS cyclotrons work with a vacuum of $1 \times 10^{-6} \mathrm{~Pa}$ and expect beam losses are less than about $150 \mathrm{~W}$ for an accelerated beam current of $8 \mathrm{~mA}$ of $\mathrm{H}_{2}^{+}$. The residual gas is assumed to be $100 \%$ hydrogen. The larger extraction radius of the single stage cyclotron means the vacuum has to be better than $8 \times 10^{-7} \mathrm{~Pa}$ in order to maintain beam losses below the $150 \mathrm{~W}$.

The third and most critical issue is the dissociation of the vibrational states contained in the $\mathrm{H}_{2}{ }^{+}$beam. The DAEסALUS solution, see Fig. 14 , is feasible also for the single stage cyclotron but it is not clear how to remove the protons from dissociated higher vibrational states, which could be more than $1 \%$ of the beam current. These will have energies up to, and possibly over, $600 \mathrm{MeV}$ and will almost surely be lost inside the acceleration chamber. 


\section{Fixed Field Alternating Gradient Accelerators}

The drive for high beam power in the $\mathrm{GeV}$ range, high duty cycle or $\mathrm{CW}$ operation, reliability and precisely controlled beams with affordable technology and footprint has resurrected interest in Fixed-field Alternating Gradient Accelerators (FFAG). FFAG accelerators potentially support repetition rates in the $\mathrm{kHz}$ range like synchrocyclotrons, with further potential for $\mathrm{CW}$ acceleration recently demonstrated. They have large geometrical and momentum acceptances, and also embody advantages of the synchrotron such as strong focusing, which mitigates space charge effects and minimizes beam losses. With features of the cyclotron and synchrotron combined, FFAGs have been subject to renewed attention beginning in the late 1990s [39], with on-going $R \& D$ at international laboratories and universities, actively exploring new directions in accelerator science, in concept and in application.

\subsection{Introduction and history}

Following the invention of the concept in 1953, a year after the invention of strong focusing, radial and spiral FFAG optics were devised by the MURA group. A history of MURA and the early FFAG years can be found in [40]. Three electron prototypes, the third and last one a $50 \mathrm{MeV}$, twoway ring, were designed and operated between 1954 and 1962. All three were based on sector magnets (radial FD doublet for the first and third, spiral sector for the second one [40]) with magnetic fields following the characteristic scaling law:

$$
B \propto r^{k}
$$

where $r$ is the radius and $\mathrm{k}$ a constant field index. They were called "scaling" FFAGs, by reference to the geometrically similar orbit shapes scaling with energy. Optics and tunes remain unchanged along the acceleration ramp as a consequence.

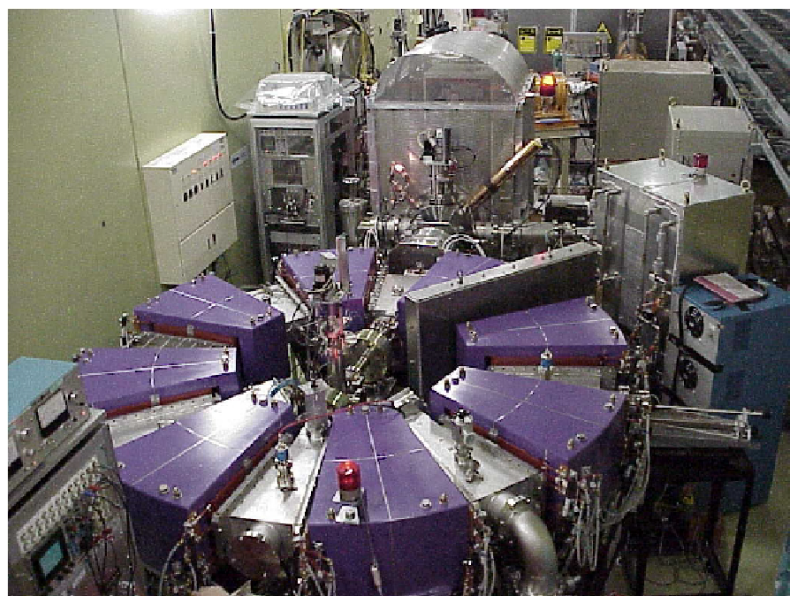

Fig. 24. Proof-of-principle (PoP) $500 \mathrm{keV}, 2.5 \mathrm{~m}$ diameter, proton scaling FFAG at KEK.

During the same period, vertical FFAGs were proposed [41], and subjected to detailed studies [42]. Synchrotrons eventually won in the quest for high energy beams. FFAG activity remained dormant until the late 1990s, when scaling FFAGs were proposed for fast acceleration of muons in the neutrino factory, and for the $4 \mathrm{MW}$ proton driver for muon production [43]. A low energy proof-of-principle proton demonstration machine was built [44], see Fig. 24.

In parallel, the concept of "non-scaling FFAG", based on strong focusing linear optics (essentially, combined function or quadrupole magnets), was proposed [45], for rapid acceleration of large geometric and momentum emittance (short-lived) muon beams. This latter concept was realized in a proof of principle machine, EMMA, at Daresbury laboratory [46]. Investigation of its potential for the design of isochronous or quasi-isochronous high power proton beam lattices has been a focus in recent years [47, 48, 49, 50]. In an isochronous machine $\gamma$ equals transition- $\gamma$, and longitudinal space charge cannot debunch the beam [51], whereas strong focusing alleviates space charge effects thereby maintaining small beam emittance and reducing losses, a particularly serious problem at the high powers required for ADS. 


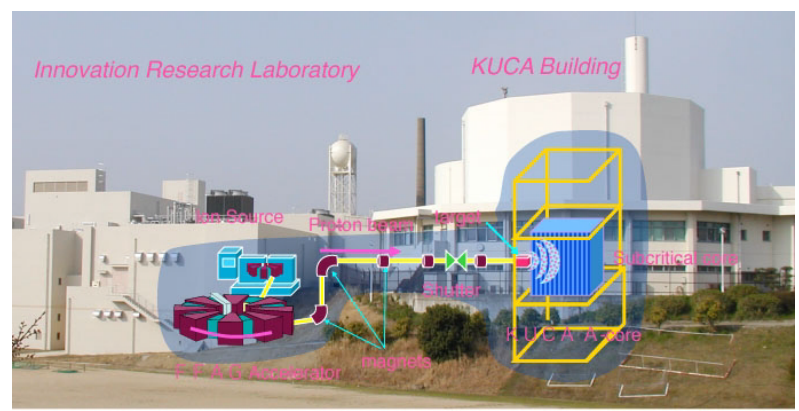

Fig. 25. KURRI-KUCA ADS-Reactor experiment using an FFAG proton driver (to the left) delivering beam to an experimental reactor core (to the right).

FFAG design studies have profited from computation advances, both in algorithms and in performance. Calculations of 3-D field maps of scaling, radial, spiral and other complex gradient dipole magnets, and tracking of accelerated 6-D bunches as they spiral outward with energy through those maps can be both performed. Design fields and corresponding magnet profiles can be efficiently optimized. Program development specific to FFAG design and simulation is an active field, and includes space charge for the high power sector, for example OPAL [32,50]. FFAG designs track accelerator technologies, exploring the latest in superconducting magnet designs and high gradient RF systems, which are particularly relevant to the ADS-Reactor application.

A comprehensive review of the field in 2007 can be found in [52]. The dominant innovations and promising FFAG designs and machines in the more recent period, from 2007 to 2015 , will be presented here with particular details on ADS-specific studies.

\section{Scaling FFAG}

\subsection{KURRY-KUCA experiment}

Strong focusing and very large acceptances with fast acceleration characterize the FFAG technology in the design of a proton driver, in addition to the acceleration of short-lived, large emittance muon beams, for the Neutrino Factory. The first proton FFAG, a $500 \mathrm{keV}$ proof-of-principle radial sector (PoP), was built, and operated at KEK in

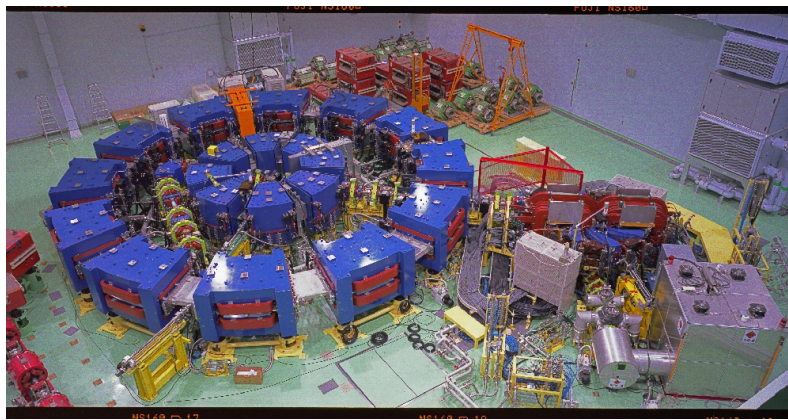

Fig. 26. KURRI-KUCA $150 \mathrm{MeV}$ radial sector scaling FFAG proton driver.

December 1999 [44], see Fig. 24. The cell structure was innovative and based on a DefocusingFocusing-Defocusing (DFD) dipole triplet cell that allowed insertion of longer straight sections compared to an FD doublet. Following success at $500 \mathrm{keV}$, a $150 \mathrm{MeV}$ FFAG was constructed at KEK in the early 2000s, in a medical R\&D program framework. This machine came into operation in 2003 [53]. The $150 \mathrm{MeV}$ design became the template for the ADS-Reactor R\&D installation for the Kyoto University Critical Assembly (KUCA) at the Kyoto University Research Reactor Institute (KURRI) in the mid2000s, as shown in Fig. 25. Importantly, this facility demonstrated chained FFAGs: a $2.5 \mathrm{MeV}$ spiral FFAG based on parallel-gap, coil-shaping magnets and betatron acceleration, first of the kind. This injects into a $20 \mathrm{MeV}$ ring similar to the $150 \mathrm{MeV}$ ring, with all three stages visible on Fig. 26. First coupling of the KUCA FFAG to the subcritical core was achieved in March 2009, with a $100 \mathrm{MeV}$ beam from the high-energy stage.

Thorium-loaded ADS-R experiments were performed in March 2010 using this $100 \mathrm{MeV}$ beam and a repetition rate of $30 \mathrm{~Hz}$ producing $5 \mathrm{~mW}$ beam power. Planned upgrades include the production of higher beam energy, in the 150$700 \mathrm{MeV}$ range, based on an additional spiral lattice FFAG stage (see next section), allowing a neutron flux increase of a factor of 30 . On-going R\&D at the KURRI-KUCA installation includes charge-exchange injection in the $150 \mathrm{MeV}$ ring, using an $11 \mathrm{MeV} \mathrm{H}^{-}$injector linac, with the aim of 


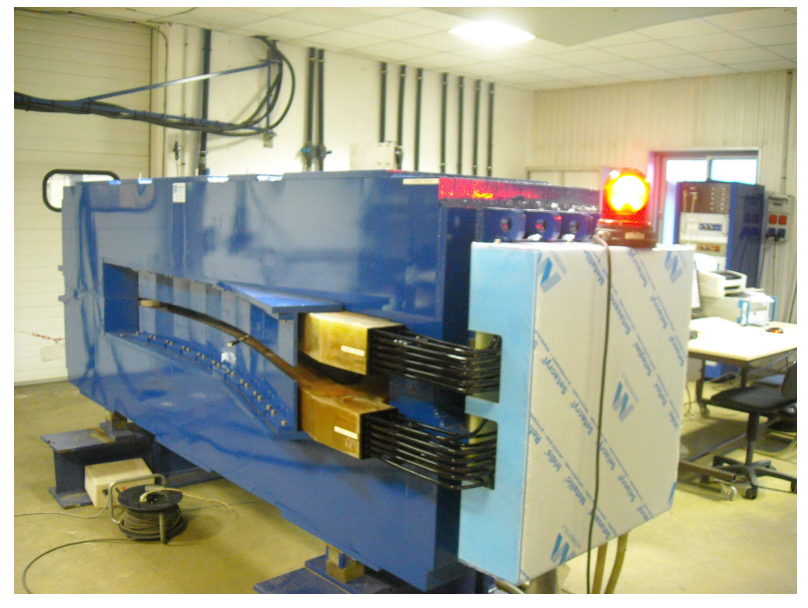

Fig. 27. A gap shaping, spiral sector scaling FFAG magnet, first of its kind [55].

reaching current of 10 's of $\mu \mathrm{A}$ as part of the roadmap toward high power studies [54].

\subsection{Exploring further}

Medical applications foster lattice and technological R\&D in the 200 to $300 \mathrm{MeV}$ range. An example is the 10-cell, spiral lattice, $200 \mathrm{MeV}$ proton FFAG designed in the 2005-2009 [55]. Fabrication and magnetic measurement of a fullscale spiral sector scaling magnet was part of the project, as shown in Fig. 27. It validated the design optical parameters of the spiral, gap shaping magnet technology, including workarounds to compensate the vertical tune variation with radius as induced by the varying gap. Lesson learned in that magnet design are further exploited in the $700 \mathrm{MeV}$ ring planned for the upgrade of the KURRI-KUCA installation [56].

\section{Non-Scaling FFAG}

A new concept was introduced in the late 1990s, in the frame of the Neutrino Factory R\&D [52], that of linear FFAG optics, called "non-scaling" since the property of scaling orbits is lost. In this novel optics the cell magnets are combined function dipoles or radially displaced quadrupoles [57].

Due to the small dispersion function, "nonscaling" FFAG optics allows far more compact and narrower magnet arrangements than scaling optics.

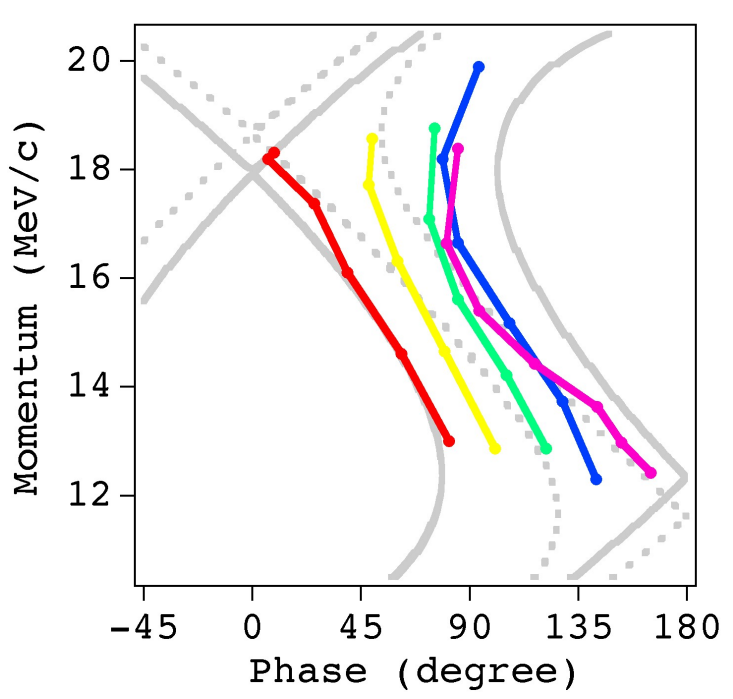

Fig. 28. A measurement of beam acceleration along the serpentine channel at EMMA. The theoretical Hamiltonian path system is shown for comparison.

These optics are quasi-isochronous in the ultrarelativistic regime, namely $\gamma_{\mathrm{tr}}$ can be made close to $\gamma$ at all energies, allowing fixed RF frequency acceleration, a method known as "serpentine acceleration" due to the shape of the longitudinal phase space separatrix system in this acceleration mode [58], see Fig. 28.

Variants include "minimal emittance" lattices that minimize the dispersion function (as in "minimal emittance" light-source optics), and minimize the radial extent of the magnets [59]. Since the magnets operate at fixed field, their strength decreases with beam energy, and cell tunes decrease as a consequence during acceleration ramp, typically in the range from $\mathrm{Q}_{\mathrm{x}, \mathrm{y}}=0.4$ to 0.1 . Further development of quasi-isochronous FFAG optics variants, include cells based on nonlinear magnets, for muon acceleration and multi-GeV proton driver application [48], as is addressed below. Note that acceleration in linear-field nonscaling FFAGs can only cover a factor of about 4 in momentum, before the tunes become unstable. Later applications of non-rectangular pole faces and higher-order fields increase the acceleration range up to an order of magnitude, this is discussed further in section 8.3. This is an important point for ADS which requires a large acceleration range. This linear FFAG lattice concept is being exploited 
in the design of eRHIC, an electron-ion collider project, in which an energy-recovery electron recirculating linac has arc designs based on such optics [60].

\subsection{The EMMA experiment}

An "Electron Model for Many Applications" (EMMA) was built in the framework of an international collaboration, to demonstrate both the non-scaling FFAG concept and the serpentine quasi-isochronous acceleration across betatron resonances [46]. EMMA is comprised of 42 quadrupole doublet cells, for a circumference of $16 \mathrm{~m}$, with acceleration provided by $19 \mathrm{RF}$ cavities evenly placed around the ring. Construction of the EMMA ring at Daresbury started in 2007. Commissioning was performed in 2010, and rapid, quasi-isochronous acceleration in the serpentine channel was demonstrated in 2011, see Fig. 28.

\subsection{The Proton driver lattice}

High power rings were initially derived based on the linear FFAG concept [52]. However the low-medium relativistic dynamics precluded quasiisochronous acceleration, so these used pulsed, frequency-swept RF and tuned cavities. These cavities permit repetition rates of up to a $\mathrm{KHz}$ but even this rate imposes a strict limit of 1-2 MW in the $\mathrm{GeV}$ proton beam power achievable. Continuous wave acceleration based on "harmonic number jump" using fixed frequency RF systems was also investigated but was found to be technically difficult, considering the RF voltage range required [47]. This led to research into the possibility of enabling isochronous orbits in an FFAG by combining a nonlinear field profile in the magnetic components coupled to an adaptive edge contour. This approach has produced models currently under study for the ADS application.

\subsection{Isochronous high energy FFAGs}

Isochronous and quasi-isochronous non-scaling FFAG optics have been under investigation since the mid-2000's. Isochronism allows on-crest acceleration with the highest plug-to-beam power
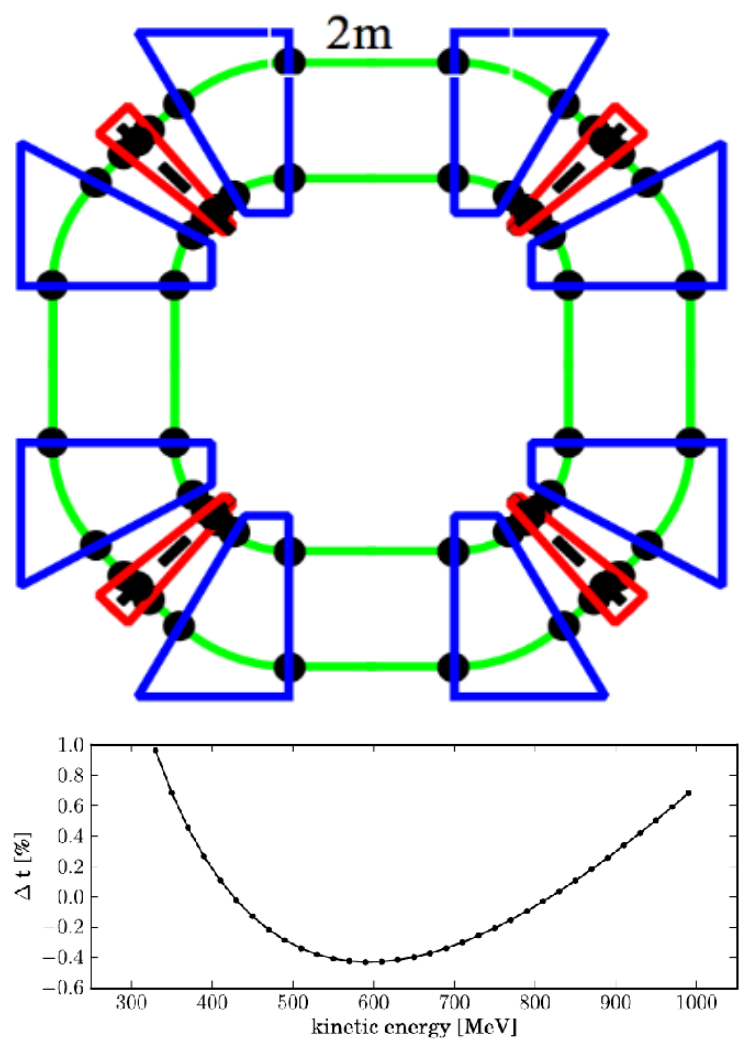

Fig. 29. On the top: Principle lattice of a quasi-isochronous, quasi-constant tunes, 6-cell $0.33-1 \mathrm{GeV}$ FFAG ring, $7 \mathrm{~m}$ radius, based on non-linear radial field profiles with alternating normal (blue) and reverse-gradient (red) components. On the bottom: Typical energy dependence of the time-of-flight.

efficiency, as well as the capability for $\mathrm{CW}$ beam delivery minimizing bunch charge and associated space charge effects. CW operation is considered at this time the only viable path to a $10 \mathrm{MW}$ class FFAG accelerator. In a 2010 White Paper on accelerator technologies for Accelerator Driven Systems [61], FFAG accelerators were described as "promising ... albeit without the capability for true CW operation". Since then, significant progress has been made on high-power, $1-\mathrm{GeV}$ isochronous FFAG designs. Two advancements make these new designs a viable option for high power proton drivers: small tune variation over the acceleration cycle (avoiding crossing betatron resonances), combined with nearly isochronous acceleration; i.e. a fixed-field, fixed-RF $\mathrm{CW}$ accelerator with synchrotron-like stable tunes. These goals are 
achieved by tailoring an arbitrary radial field profile, possibly matched to an optimized linearedge contour, independently varied between normal and reversed field components. Some of the studies performed are recalled as an illustration in the following.

In the mid-2000s a symmetric cell design based on strongly non-linear dipoles allowed multi $\mathrm{GeV}$ muon and MW class proton driver lattices [48]. The additional optical variables introduced by departing from the linear optics and by the fivemagnet cell yielded sufficient flexibility to achieve isochronous or quasi-isochronous optics in addition to strong focusing and small horizontal dispersion (meaning small radial magnet extent). Ring layouts allowed high power beam handling, including matched insertion straights for easier injection, acceleration, extraction, space for beam-loss collimators, and multiple-cell cavities to reduce the number of RF systems. However beam energy in this design is higher than required for ADS and lower energy stages have not been worked out. A reduced repetition rate, $50 \mathrm{~Hz}$, was foreseen in addition in muon production application.

More recently, DFD triplet cell designs based on nonlinear radial field profiles achieve nearly isochronous orbits while minimizing the tune variation. Fig. 29 displays a typical four-cell lattice, a representative model for beam dynamics studies in the range from $0.3 \mathrm{GeV}$ to $1 \mathrm{GeV}[49,50]$. The lattice includes long drift sections for beam manipulations, control and acceleration. The lattice incorporates wedge-shaped magnets with a radial magnetic field dependence in order to produce stable tunes and to achieve a time-of-flight variation that is within $\pm 0.5 \%$ over the full acceleration range. Multi-turn beam stability along with error tolerances have been studied in these lattices. Large dynamic apertures of tens of thousands mm.mrad horizontal, and of the order of $100 \mathrm{~mm} . \mathrm{mrad}$ vertical, can be achieved [62]. Simulations in OPAL demonstrated currents in $20 \mathrm{~mA}$ range without transverse space charge blowup, confirming the expectations from strong focusing and quasi-constant tunes.

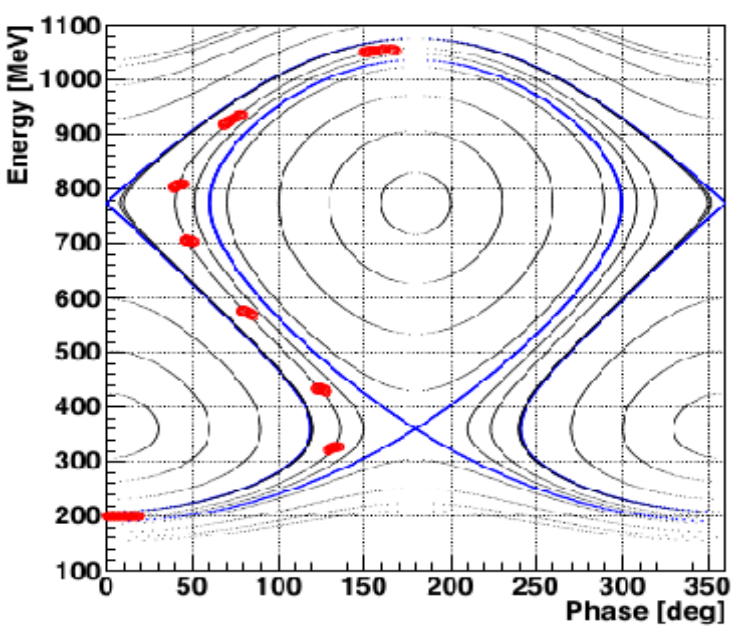

Fig. 30. Serpentine acceleration of a proton beam, from 200 to $1100 \mathrm{MeV}$, in a scaling FFAG lattice.

This design also allows multiport injection as discussed in the cyclotron section, so mitigating space charge problems in the upstream acceleration stages. Extended discussions and simulation data $[48,49]$ show this type of design to be a promising candidate for ADS. Design choices, based on radial and axial magnetic field profile methods, and the theoretical principles on which they are based, have been subject to dedicated review [63].

Medical FFAG design studies have been contributing in these researches regarding isochronous, constant tune, non-scaling FFAG optics, such as PAMELA [64].

\subsection{Serpentine Acceleration in Scaling FFAGS}

Quasi-isochronous serpentine acceleration demonstrated experimentally in EMMA, also applies to scaling FFAG optics. Indeed, fixed RFfrequency serpentine acceleration in the nonrelativistic regime was proven theoretically, recently, at Kyoto University [65], suitable for ADS-R application.

This experimental demonstration was performed with an electron ring model accelerating, from $164 \mathrm{keV}$ to $8 \mathrm{MeV}$ in an FDF scaling triplet lattice at transition gamma (764 $\mathrm{keV}$ ), at an RF frequency of $75 \mathrm{MHz}$ (harmonic 1), and $750 \mathrm{kV} /$ gap. 
A proton driver lattice has been designed, and acceleration from $200 \mathrm{MeV}$ to $1.1 \mathrm{GeV}$ is achieved, see Fig. 30, with an orbit excursion from 3 to 5.9 meters, $9.6 \mathrm{MHz}$ fixed frequency $\mathrm{RF}$ on harmonic 1 , at a rate of $15 \mathrm{MV} /$ turn.

Theoretical investigation of the parameter space in the non-relativistic serpentine acceleration mode, for high-power beam production, can be found in [66]. Use of the serpentine channel granted is under study. However, the further away from isochronism, the higher the required accelerating voltage. The typical voltage required is higher than needed in non-scaling variants, for example.

\section{Vertical FFAG}

Vertical FFAG optics represent a special area of isochronous FFAG R\&D [41,42]. Only limited exploration has been made in the ADS application up to now. The exponential variation of the vertical field component in vertical FFAGs causes the beam orbit to be an ascending helix at constant radius. They are isochronous for ultra-relativistic beams, since the vertical spiraling preserves orbit length. The principle was re-explored in the recent years, yielding various designs including a principle scheme of a quasi-isochronous non-planar spiral sector ring. In this case the optics evolves from cyclotron-type and planar orbit at lower energies, to vertical FFAG style as energy increases [67].

\section{Conclusions}

The cyclotron represents one of the oldest concepts for resonant particle acceleration and has been from the beginning proposed for the transmutation of elements. Their ability to produce high power beams is demonstrated not only by the success of the PSI and TRIUMF Cyclotrons but also from the many small- and medium-sized commercial cyclotrons used for radioisotopes production.

New simulation tools, like the OPAL code [32], theoretical investigations [16] and experimental experience with the transport and acceleration of very intense beams are the base for the next jump into the $10 \mathrm{MW}$ territory.

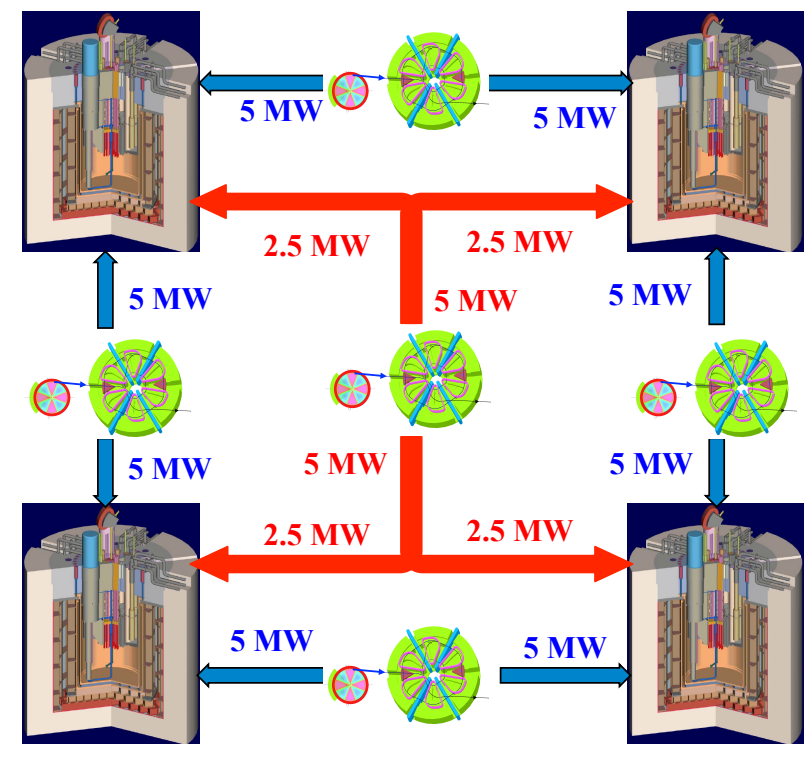

Fig. 31. Layout of a power plant with 4 subcritical reactor driven by 5 High Power Cyclotrons able to deliver 12.5 MW to each core.

The last three cyclotron proposals briefly described in section 5 are good candidates on which to base a successful ADS-R installation. Probably a new proposal based on the DAESALUS ring Cyclotron using the new concept of the fast extraction and the new technological development of the RF cavities under study at TAMU could lead to a powerful cyclotron with a conversion efficiency of wall plug power to the beam that could be near $70 \%$. Moreover the idea to use the RFQ to inject high current beam in the TAMU100 cyclotron, proposed earlier by Hamm [68], has been proposed also for the DAESALUS compact cyclotron [69].

The ADS cyclotron described in section 5 should be able to deliver a beam power of about 13 MW. For example, Fig. 29 shows a tentative solution for a 4 subcritical reactor power plant driven by 5 ADS cyclotrons. Even though each cyclotron will have reliability higher than $90 \%$, according to the experience of PSI, it is not possible to avoid shutdown for maintenance and accidental failures. The use of one extra cyclotron allows operation of each accelerator at $70 \%$ of its maximum power to increase their reliability. Moreover, in case of failure or maintenance of one cyclotron the other accelerators can quickly 
redistribute their beam power to keep the plan running at normal capacity.

Advances in isochronous and strong-focusing optics promote the FFAG technology as a potential solution for a MW class proton driver. The high reliability requirement can imply accelerator redundancy, not necessarily cost-prohibitive in the FFAG option. With multiple RF cavities, an RF trip would result in slower acceleration cycle rather than interrupting beam. Multiple injectors are supported by the long straight sections in racetrack style FFAG layouts. With redundancy in the injectors, an upstream failure of an accelerator does not interrupt beam, it essentially reduces current (the time needed until beam current in operational injectors can respond, on ultra-fast timescales) thus limiting thermal shock and avoiding damage to the reactor.

Another advantage, in the non-scaling FFAG design, is compactness and isochronism compared to the scaling versions and designs which can use room temperature magnets and still remain as compact as the proposed SC cyclotrons.

In the matter of production of multi-MW proton beams for ADS-R installations, there is still much to devise in the domain of FFAG optics and technology. Active international FFAG workshops are held yearly since the late 1990s, numerous publications can be found in PAC, IPAC and Cyclotron accelerator conferences. Topics cover compact lattices, $\beta<1$ regime, isochronous lattices and $\mathrm{CW}$ acceleration, reverse bend optics, serpentine acceleration, magnet and RF technology, superconducting components, high power beams, and applications in many domains from hadron therapy to nuclear energy.

The development of fixed-field rings is supported by active R\&D programs in several laboratories world-wide, however there is still a need to foster wider progress in the many crucial accelerator technology areas, to realize fully the advantages of this technique.

\section{Acknowledgements}

In this review it was not possible to cover all the contributions to the ADS concept. Our attention was focused on what in our opinion are the most relevant technical developments and for sure we have failed to include the contributions of many scientists that have worked in this field. We apologize for being incomplete.

The authors wish to thank our colleagues $\mathrm{M}$. Craddock, P. Mandrillon, P. Mc Intyre, Y. Mori, and $\mathrm{M}$. Seidel who provided pictures, drawings and information for this paper. In particular the authors would like to thank J. Alonso (LBNL), S. Machida (RAL), C. Johnstone (FNAL), S. Peggs (BNL), and D. Winklehner (MIT), for their contributions and for reading the manuscript.

\section{References}

[1] F. Carminati et al., "An energy amplifier for cleaner and inexhaustible nuclear energy productiondriven by a particle beam accelerator", CERN/AT/93-47 (ET).

[2] C. Rubbia et al, "Conceptual design of a fast neutron operated a high power energy amplifier", CERN/AT/95-44 (ET).

[3] C.M. van Atta, "A Brief History of MTA Project", ERDA Information Meeting on Accelerator Breeding, January 19-29 (1977).

[4] Vi. I. Goldanski, R.G. Vassylkov et al., Atomnaya Energiya 48, (1978) p. 329.

[5] C. D. Bowman et Al., Nucl. Inst. \& Meth in Phys. Res. A320 (1992) pgs. 336-367.

[6] W. Gudowski, Nucl. Phys. A654(1999) 436c-457c

[7] U. Schryber et al., www.jacow.org, Cyclotron 1995, p. 32.

[8] T. Stammbach et al., www.jacow.org, Cyclotrons 1992, p. 28.

[9] W. Joho, www.jacow.org, Cyclotron 1981, p. 337

[10] M. Seidel, www.jacow.org, IPAC 2010, p. 1309

[11] S. Henderson, "Thorium Energy Conference" ThEO 2011, New York.

[12] H. Ait Abderrahim et al., "Accelerator and Target Technology for Accelerator Driven Transmutation and Energy Production"; DoE white paper, 2010.

[13] N. Fiétier, P. Mandrillon, www.jacow.org, Cyclotrons 1995, p. 462.

[14] S. Adam, www.jacow.org, Cyclotrons 1995, p. 446.

[15] Y.J. Bi et al., "Towards Quantitative Simulation of High Power Proton Cyclotrons", Phys. Rev. ST Accel. Beam 14 (2011) 054402. 
[16] C. Baumgarten, www.jacow.org, Cyclotrons 2013, p. 315 .

[17] T. Stambach et al., "The feasibility of high power cyclotrons", NIM in Phys. Res. B, 113 (1996) p. 1.

[18] M. Craddock, "Critical Beam-Intensity Issues in Cyclotrons - Overview of the Santa Fe Workshop", www.jacow.org, Cyclotrons 1998, p. 377.

[19] U. Trinks, www.jacow.org, Cyclotrons 1992, p. 693.

[20] A. Cazan et al., www.jacow.org, Cyclotrons 1998, p. 323.

[21] L. Calabretta and D. Rifuggiato, "A Superconducting Cyclotron for $\mathrm{H}_{2}^{+}$with extraction by stripping", ECPM 31 abstract, KVI Groeningen (NL), 1997.

https://www.kvi.nl/ agorcalc/ecpm31/abstracts/abs tracts.html

[22] L. Calabretta and D. Rifuggiato, Superconducting Cyclotron for $\mathrm{H}_{2}^{+}$, www.jacow.org, Cyclotrons 1998, p. 665.

[23] N. Fiétier and P. Mandrillon, www.jacow.org, Cyclotrons 1998, p. 389.

[24] M. Reiser, Theory and design of charged Particle Beams, Wiley series in Beam Phys. And Acc. Tech.

[25] T. Mitsumoto et al., www.jacow.org, Cyclotrons 2010, p. 430.

[26] L. Calabretta et al., www.jacow.org, IPAC 2014, p. 788.

[27] J.M. Conrad and M.H. Shaevitz, Phys.Rev.Lett. 104, 141802 (2010).

[28] G. Kim et al., www.jacow.org, PAC 2001, p. 2593

[29] P. Mandrillon et al., Thorium Energy Conference 2013, CERN,

https://indico.cern.ch/event/222140/contributions

[30] L. Calabretta et al., www.jacow.org, EPAC 2000, p. 918.

[31] A. Radovinsky et al., "Engineering Study of Sector Magnet for the DAESALUS Experiment", IEEE Transactions on Applied Superconductivity, 2014, 4500204

[32] Object Oriented Particle Accelerator Library https://amas.psi.ch/OPAL.

[33] J.J. Yang et al.; Nucl. Inst. \& Meth. in Phys. Res.; section A; vol. 704; (2013) p.84-91.

[34] P. McIntyre et al ., 'Strong-Focusing Cyclotron for High-Current Applications', IPAC 2012, p 436.

[35] F.M. Russel et al., www.jacow.org, Cyclotrons 1963, CYC63H03.

[36] N. Pogue et al., www.jacow.org, IPAC 2012, p. 2354.

[37] P. Mc Intyre at al., IPAC 2015, MOPMA047.

[38] P. McIntyre et al., IPAC 2015, THPF135.

[39] M. Craddock, The rebirth of the FFAG, CERN Courier, July 27, 2004, http://cerncourier.com/cws/article/cern/29119.

[40] F. T. Cole, O Camelot, A Memoir of The MURA Years (April 1994).
[41] T. Ohkawa, Phys. Rev. 100, 1247 (1955).

[42] G. Leleux et al., FFAG helicoidal, Rapport O.C. 70, CEA/SPA, Saclay (19 juin 1959).

[43] Y. Mori, www.jacow.org, EPAC 2002, p. 278.

[44] Y. Mori, CERN Courier, Sept. 21, 2000, $\mathrm{http} / / /$ cerncourier.com/cws/article/cern/28304.

[45] F. Mills, C. Johnstone, Proc. 4th Int. Conf. Physics Potential and Development of $\mu+\mu$ - Colliders, San Francisco, 693-698 (1998).

[46] S. Machida et al., Acceleration in the linear nonscaling fixed-field alternating-gradient accelerator EMMA, Nature Physics, Jan. 2012.

[47] A.G. Ruggiero, FFAG-Based High-Intensity Proton Drivers, Proceedings of the ICFA-HB2004 Workshop, Bensheim, Germany, Oct. 18-22, 2004.

[48] G. Rees, D. Kelliher, New, high power, scaling, FFAG driver ring designs, Proceedings of HB2010, Morschach, Switzerland.

[49] C. Johnstone et al, $1 \mathrm{GeV}$ CW Nonscaling FFAG for ADS and Magnet Parameters, Proceedings of the IPAC, pgs. 4118-4020, May 20-25, New Orleans, LA 2012.

[50] S. Sheehy, The potential for a high power FFAG proton driver for ADS, arXiv:1310.3588v1 [physics.acc-ph] 14 Oct. 2013.

[51] R. Baartman, S. Martin, FFAGs and Cyclotrons, A summary of the session on FFAGs and Cyclotrons, ICFA-HB2004 Workshop, Bensheim, Germany, Oct. 18-22, 2004.

[52] C.Prior, Editor, FFAG accelerators, ICFA BD Newsletter 43, pp. 14-133, Aug. 2007.

[53] Y. Yonemura et al., www.jacow.org, PAC 2005, p. 1943.

[54] Y. Ishi, KURRI R\&D, Status, FFAG 2014 workshop, Brookhaven National Lab., Sept. 22-26, 2014.

[55] S. Antoine et al., NIM A 602 (2009) 293-305.

[56] B. Qin, Y. Mori, NIM A A 648 (2011) 28-34.

[57] C. Johnstone et al., www.jacow.org, PAC 1999, p. 3068.

[58] S. Koscielniak, C. Johnstone, Proc. Snowmass 2001, T508 (2001).

[59] D. Trbojevic et al., FFAG lattice without opposite bends, Neutrino Factory Workshop, 27 Sep-1 Oct 1999, AIP Conf. Proc. 530, 333 (2000).

[60] D. Trbojevic, FFAG Lattice Design of eRHIC and LHeC, EIC14 Workshop, Jefferson Lab., April 2014. https://www.jlab.org/conferences/eic2014/

[61] H. Aït Abderrahimh et al., Accelerator and Target Technology for Accelerator Driven Transmutation and Energy Production, US DOE, September 17, 2010.

[62] R. Appleby et al., The Dynamics of Ultra-compact and Isochronous GeV Energy FFAGs, Proceedings of the International Particle Accelerator Conference, Dresden Germany, pgs 780-783, Jun 15-20, 2014. 
[63] M. Craddock, Isochronous radial-sector nonscaling FFAGs using different $\mathrm{F}$ and $\mathrm{D}$ field profiles, FFAG 2014 Workshop, Brookhaven National Laboratory. https://indico.bnl.gov/conferenceDisplay.py?confI $\mathrm{d}=686$

[64] K. Peach et al., PAMELA overview and status, Proceedings of the 2010 International Particle Accelerator Conference, Kyoto, 2010.

[65] E. Yamakawa et al., Serpentine acceleration in zero-chromatic FFAG accelerators, NIM A 716 (2013) 46-53.

[66] S. Koscielniak, Optimum serpentine acceleration in scaling FFAGs, FFAG'13 workshop, TRIUMF, 2013.

[67] S.J. Brooks, Vertical Orbit Excursion Fixed Field Alternating Gradient Accelerators, Phys. Rev. ST Accel. Beams 16, 084001 (2013).

[68] R. Hamm et al., www.jacow.org, Cyclotrons 1981, p. 359.

[69] D. Winklehner et al. www.jacow.org, IPAC 2015, WEPTY048. 